\title{
Bottom-quark effects in Higgs production at intermediate transverse momentum
}

\author{
Fabrizio Caola, ${ }^{a}$ Jonas M. Lindert, ${ }^{a}$ Kirill Melnikov, ${ }^{c}$ Pier Francesco Monni, ${ }^{b}$ \\ Lorenzo Tancredi ${ }^{b}$ and Christopher Wever ${ }^{c, d}$ \\ ${ }^{a}$ Institute for Particle Physics Phenomenology, Durham University, \\ Durham DH1 3LE, U.K. \\ ${ }^{b}$ TH Division, Physics Department, CERN, \\ CH-1211 Geneva 23, Switzerland \\ ${ }^{c}$ Institute for Theoretical Particle Physics (TTP), \\ KIT, Karlsruhe, Germany \\ ${ }^{d}$ Institut für Kernphysik, KIT, \\ 76344 Eggenstein-Leopoldshafen, Germany \\ E-mail: fabrizio.caola@durjam.ac.uk, pier.monni@cern.ch, \\ jonas.m.lindert@durham.ac.uk, kirill.melnikov@kit.edu, \\ lorenzo.tancredi@cern.ch, christopher.wever@kit.edu
}

AbStract: We provide a precise description of the Higgs boson transverse momentum distribution including top and bottom quark contributions, that is valid for transverse momenta in the range $m_{b} \lesssim p_{\perp} \lesssim m_{t}$, where $m_{b}$ and $m_{t}$ are the bottom and top quark masses. This description is based on a combination of fixed next-to-leading order (NLO) results with next-to-next-to-leading logarithmic (NNLL) transverse momentum resummation. We show that ambiguities in the resummation procedure for the $b$-quark loops are of the same order as the related fixed-order uncertainties. We conclude that the current uncertainty in the top-bottom interference contribution to the Higgs transverse momentum spectrum is $\mathcal{O}(20 \%)$.

KeYwords: NLO Computations, QCD Phenomenology

ARXIV EPRINT: 1804.07632 


\section{Contents}

1 Introduction 1

2 Resummation of the Higgs transverse momentum distribution 4

2.1 The standard point-like case 4

2.2 Issues with $b$ quarks 8

3 Results 11

3.1 Inclusion of bottom-quark loops and matching uncertainties 11

$\begin{array}{lll}3.2 & \text { Mass-scheme uncertainty and final results } & 15\end{array}$

$\begin{array}{lll}4 & \text { Conclusions } & 17\end{array}$

A Resummation and matching: details $\quad 19$

$\begin{array}{lll}\text { A.1 The LL case } & 19\end{array}$

A.2 Final formulas for NNLL resummation 24

$\begin{array}{lll}\text { A.3 Matching to fixed order } & 27\end{array}$

\section{Introduction}

Understanding the Higgs particle observed at the LHC requires studies of its properties that include quantum numbers and couplings to gauge and matter fields. Current experimental results have relatively small $\mathcal{O}(10 \%-20 \%)$ uncertainties in Higgs couplings to electroweak gauge bosons and larger $\mathcal{O}(100 \%)$ uncertainties in Higgs Yukawa couplings, especially for light quarks [1]. However, it is quite conceivable that physics beyond the Standard Model manifests itself in smaller, few percent, contributions to Higgs couplings. Thus, facilitating further improvements in extracting Higgs couplings to gauge bosons and helping constrain Yukawa couplings are very important issues in contemporary Higgs physics.

Both of these issues can, at least partially, be addressed by improving the description of Higgs boson production in gluon fusion. Indeed, since $g g \rightarrow H$ is the main production mechanism of Higgs bosons at the LHC, a refined understanding of this process in QCD perturbation theory will lead to an improved understanding of fiducial cross sections and, eventually, will allow for a better extraction of various Higgs couplings constants from e.g. Higgs decays to bosonic final states.

Although the contributions of bottom and charm loops to the $g g H$ coupling and direct production of a Higgs boson in quark fusion $q \bar{q} \rightarrow H, q \in\{c, b\}$ are small in the Standard Model, if the Yukawa couplings differ from their Standard Model values, these light-quark effects in Higgs production become much more important. In fact, it was pointed out $[2,3]$ that studies of kinematic distributions of Higgs bosons produced in hadron collisions may lead to interesting constraints on light quark Yukawa couplings, especially at the highluminosity LHC. 
A particularly important and highly non-trivial kinematic distribution is the Higgs boson transverse momentum spectrum. At the LHC, Higgs bosons are produced with very different transverse momenta, from very small to very large; the $p_{\perp}$ distribution peaks at $p_{\perp} \approx 15 \mathrm{GeV}$. Depending on the value of the Higgs transverse momentum, the $p_{\perp}$ distribution is sensitive to different physics, from multiple emissions of soft gluons at small $p_{\perp}$ to top quark mass effects at the tail of the spectrum. The difficulty in describing the Higgs transverse momentum distribution as a whole is related to this point.

Higgs production in gluon fusion receives contributions from top and light-quark loops. Since Yukawa couplings are proportional to quark masses, top quark loops play the dominant role. For values of the transverse momenta $p_{\perp} \lesssim m_{t}$, top loops can be treated in the $m_{t} \rightarrow \infty$ approximation. This leads to enormous technical simplifications since, essentially, it allows us to "remove" one loop from the computations that involve the $g g H$ vertex. As the result, the $m_{t} \rightarrow \infty$ approximation allowed for the computation of next-to-next-tonext-to-leading order $\left(\mathrm{N}^{3} \mathrm{LO}\right)$ QCD corrections to the inclusive cross section and basic kinematic distributions [4-6], as well as next-to-next-to-leading order (NNLO) QCD corrections to the production of Higgs bosons in association with one jet [7-11]. It is also quite straightforward to compute the $\mathcal{O}\left(1 / m_{t}\right)$ corrections to the $m_{t} \rightarrow \infty$ approximation; they are available for the total cross section [12-14] and for the Higgs $p_{\perp}$ distribution [15-17]. NLO QCD corrections including the top-quark dependence in the full Standard Model have become available recently either via a high-energy expansion $[18,19]$ or a direct numerical calculation [20] of the relevant two-loop virtual amplitudes.

When small transverse momenta $p_{\perp} \ll m_{H}$ are considered, radiative corrections to Higgs production become enhanced by large logarithms $\ln m_{H} / p_{\perp}$. It is possible to resum these logarithms in case of Higgs production in gluon fusion if the $g g H$ coupling is pointlike, which is the case in the $m_{t} \rightarrow \infty$ approximation. Such resummations were performed with ever increasing accuracy through the years [21-24]. The necessary ingredients to compute the next-to-next-to-next-to-leading logarithmic $\left(\mathrm{N}^{3} \mathrm{LL}\right)$ corrections, apart from the four-loop cusp anomalous dimension, were obtained in refs. [25-28]. This allowed for a description of the Higgs boson $p_{\perp}$ spectrum at $\mathrm{N}^{3} \mathrm{LL}+\mathrm{NNLO}[29] .{ }^{1}$

Resummed computations are usually extrapolated from small transverse momenta, where they are valid, to large momenta, where they are matched to fixed-order computations. As we explained in the previous paragraphs, the accuracy of both resummed and fixed-order computations has been constantly increasing; as a result, the $m_{t} \rightarrow \infty$ top-loop mediated contribution to the Higgs transverse momentum distribution is currently known with a precision of about $10-15$ percent for all values of the Higgs $p_{\perp}[7-11,29]$.

Having reached this level of understanding in the $m_{t} \rightarrow \infty$ limit, it is essential to ask if additional small effects, that could have been neglected previously, need to be accounted for at the present level of accuracy and, if so, if they are sufficiently well understood. Examples of such contributions are corrections to the $g g H$ interaction vertex due to light quarks and electroweak corrections to the Higgs transverse momentum distribution. Both of these effects appear at the one-loop level; light-quark contributions change the Higgs

\footnotetext{
${ }^{1}$ The numerical impact of the four-loop cusp anomalous dimension is expected to be small [30].
} 
boson transverse momentum distribution by about $-5 \%$. For moderate values of transverse momenta, electroweak contributions to the Higgs $p_{\perp}$ spectrum are smaller [31] and we neglect them in what follows.

Since QCD corrections in $g g \rightarrow H$ are known to be large for the top quark contribution, it becomes important to understand if a similar enhancement exists for light quark contributions as well. Unfortunately, such computations require two-loop calculations with massive internal particles that are currently hardly feasible. An alternative possibility is to compute the corresponding two-loop amplitudes in the approximation where all kinematic variables and the mass of the Higgs boson are considered large relative to the quark mass $m_{q}$. In this case, one computes a two-loop amplitude as an expansion in $m_{q}^{2} / m_{H}^{2}, m_{q}^{2} / s, m_{q}^{2} / p_{\perp}^{2}$. In this approximation, the relevant amplitudes have been computed in refs. [32, 33]. For $b$-quark loops, such an expansion is valid for transverse momenta larger than $\mathcal{O}(10-20) \mathrm{GeV}$ since corrections to the approximate result for two-loop amplitudes scale like $\left(m_{b} / p_{\perp}\right)^{2} \sim 0.2$ for $p_{\perp}=10 \mathrm{GeV}$.

Light-quark contributions develop a peculiar double-logarithmic dependence on the light quark masses $\ln ^{2}\left(m_{H} / m_{q}\right), \ln ^{2}\left(p_{\perp} / m_{q}\right)$. Such dependences originate from soft quark exchanges in the loops that facilitate the $g g H$ couplings. For the processes $g g \rightarrow H+g, q g \rightarrow H q$ and $q \bar{q} \rightarrow H+g$ these terms are sensitive to gluons emitted from both "inside" and "outside" the loops at finite transverse momentum, i.e. to the structure-dependent radiation.

Light-quark contributions to Higgs production in gluon fusion make the resummation of the transverse-momentum distribution difficult $[34,35]$. Indeed, since both top and bottom quark loops contribute to the $g g H$ coupling and since these loops are characterized by very different intrinsic scales for the structure-dependent radiation $\left(m_{b}\right.$ and $\left.m_{t}\right)$, it appears that one will have to treat them differently. However, this is not possible since the dominant contribution is given by the interference of the two amplitudes. In addition, since it is not understood how to resum the potentially large logarithms $\log \left(p_{\perp} / m_{b}\right)$ that appear in the light-quark loops, it becomes impossible to treat all the different contributions to the Higgs $p_{\perp}$ spectrum on the same footing. The best thing that one can do is to employ a variety of prescriptions for combining light quark contributions with small- $p_{\perp}$ resummations and to study how the resulting uncertainty in predictions compares with other sources of theoretical error.

The goal of this paper is to study the Higgs $p_{\perp}$ spectrum including top and bottomquark contributions at next-to-leading order combined with next-to-next-to-leading logarithmic transverse momentum resummation (NLO+NNLL). A similar study at leading order combined with next-to-leading logarithmic resummation (LO+NLL) was performed in ref. [34]. ${ }^{2}$ To this end, we include the recently computed NLO QCD corrections to light-quark contributions to Higgs production in gluon fusion $[32,33,36]$. We find that the uncertainty in our matched NLO+NNLL result for the top-bottom interference contribution to the Higgs transverse momentum distribution in the region $10 \mathrm{GeV} \lesssim p_{\perp} \lesssim 100 \mathrm{GeV}$

\footnotetext{
${ }^{2}$ Note that this was referred to as NLO+NLL in this reference, while we always use the formal accuracy of the differential distribution for the fixed order. In the notation of ref. [34] our result would be NNLO+NNLL.
} 
is dominated by ambiguities in the perturbative description of light-quark loops rather than by uncertainties in the resummation itself. In particular, we do not find large uncertainties related to the choice of the resummation scale for the $b$-quark loops.

The paper is organized as follows. In section 2 we briefly review the structure of small$p_{\perp}$ resummation for the case of point-like interactions, and elucidate its main assumptions and limitations. We also study light-quark contributions, discuss why in this case the resummation is challenging and describe a possible pragmatic solution to this problem. In section 3, we explain the implementation of the resummation procedure for the $b$-quark contribution and study its ambiguities, and we present our main results for the Higgs transverse momentum distribution. We conclude in section 4. Some useful formulas and derivations are collected in the appendix.

\section{Resummation of the Higgs transverse momentum distribution}

\subsection{The standard point-like case}

We would like to describe the transverse momentum distribution of Higgs bosons produced in hadron collisions. This is non trivial and requires a combination of fixed order and resummed perturbative calculations. Indeed, depending on the value of the Higgs boson transverse momentum, we can distinguish two regions. For large values of transverse momenta $p_{\perp} \sim m_{H}$, one can compute $\mathrm{d} \sigma / \mathrm{d} p_{\perp}$ in a perturbative expansion in $\alpha_{s}$ following standard rules of perturbative Quantum Field Theory. For small values of the transverse momentum $p_{\perp} \ll m_{H}$, the situation is different since emerging large logarithms $\ln \left(p_{\perp} / m_{H}\right) \gg 1$ may compensate the smallness of the strong coupling constant, $\alpha_{s} \ln ^{2}\left(p_{\perp} / m_{H}\right) \sim 1$, and spoil a conventional perturbative expansion. To deal with this case, one resums the logarithmically enhanced terms to all orders in the coupling constant, and develops a perturbative expansion on top of the resummed result.

Since, eventually, we need to describe the Higgs boson $p_{\perp}$ distribution for all values of transverse momenta, the two distinct approaches - resummation and fixed order computations - have to be combined. This is done by smoothly interpolating between results derived at small and large $p_{\perp}$. The region where the transition happens is characterized by a quantity that we refer to as the resummation scale $Q$. This scale has the following physical meaning: for $p_{\perp} \lesssim Q$, the transverse momentum distribution is mostly described by the resummed result, while for $p_{\perp} \gtrsim Q$ it is mostly described by the fixed order computation.

In order to discuss these concepts more precisely, we consider the all-order resummation in a toy model, where we work at leading-logarithmic (LL) accuracy. To this end, we consider the cumulative distribution

$$
\Sigma\left(p_{\perp}\right)=\int_{0}^{p_{\perp}} \mathrm{d} p_{\perp}^{\prime} \frac{\mathrm{d} \sigma}{\mathrm{d} p_{\perp}^{\prime}} .
$$

At low $p_{\perp}$, we resum the logarithms of $\ln p_{\perp} / m_{H}$ and write

$$
\Sigma\left(p_{\perp}\right)=\Sigma^{\mathrm{resum}}\left(p_{\perp}\right), \quad p_{\perp} \ll m_{H} .
$$


In this region, the distribution is dominated by the emission of soft and collinear partons. In the LL approximation it is sufficient to consider the most singular contribution to the QCD matrix elements, where all final-state partons are soft and strongly ordered in angle. In this limit, the squared matrix element for the emission of $n$ extra partons $g g \rightarrow H+n$ is given by the product of the matrix element for $g g \rightarrow H$ times $n$ independent eikonal factors. More specifically, at LL the partonic $p_{\perp}$ distribution

$$
\begin{aligned}
\frac{\mathrm{d} \hat{\sigma}}{\mathrm{d} p_{\perp}}= & {\left[d p_{H}\right]\left(\prod_{i=1}^{n}\left[d k_{i}\right]\right)\left|\mathcal{M}\left(p_{1}+p_{2} \rightarrow H+n\right)\right|^{2} } \\
& \times \delta^{(4)}\left(p_{1}+p_{2}-p_{H}-\sum_{i=1}^{n} k_{i}\right) \delta\left(p_{\perp}-\left|\sum_{i=1}^{n} \vec{k}_{\perp i}\right|\right)
\end{aligned}
$$

can be simplified as

$$
\begin{aligned}
\frac{\mathrm{d} \hat{\sigma}}{\mathrm{d} p_{\perp}} \simeq & {\left[d p_{H}\right]\left|\mathcal{M}\left(p_{1}+p_{2} \rightarrow H\right)\right|^{2} \delta^{(4)}\left(p_{1}+p_{2}-p_{H}\right) } \\
& \times \frac{1}{n !} \prod_{i=1}^{n}\left[d k_{i}\right]\left|M_{\mathrm{soft}}\left(k_{i}\right)\right|^{2} \delta\left(p_{\perp}-\left|\sum_{i=1}^{n} \vec{k}_{\perp i}\right|\right),
\end{aligned}
$$

where $\left[d k_{i}\right],\left[d p_{H}\right]$ are the phase space volumes of the $i$-th parton $k_{i}$ and the Higgs boson, and $M_{\text {soft }}$ is the matrix element of the single-emission eikonal current. Note that the reduced matrix element $\mathcal{M}\left(p_{1}+p_{2} \rightarrow H\right)$ is evaluated at zero transverse momentum. ${ }^{3}$

Starting from eq. (2.4) it is possible to show (for details see appendix A) that the resummed cross section takes the form

$$
\Sigma^{\text {resum }}\left(p_{\perp}\right)=\sigma_{0} e^{-\int[d k]\left|M_{\text {soft }}(k)\right|^{2}} \sum_{n=0}^{\infty} \frac{1}{n !} \prod_{i=1}^{n} \int\left[d k_{i}\right]\left|M_{\text {soft }}\left(k_{i}\right)\right|^{2} \Theta\left(p_{\perp}-\left|\sum_{i=1}^{n} \vec{k}_{\perp i}\right|\right),
$$

where $\sigma_{0}$ is the Born cross section for $g g \rightarrow H$. The overall exponential factor contains the all-order effects of soft-collinear virtual gluons which are encoded in the leading divergence of the gluon form factor $\mathcal{M}\left(p_{1}+p_{2} \rightarrow H\right) .{ }^{4}$ The distribution in the small $p_{\perp}$ region is governed by two competing mechanisms. In the strict limit $p_{\perp} \rightarrow 0$, the dominant contribution comes from emissions with finite transverse momentum $p_{\perp} \ll k_{\perp i} \ll m_{H}$ that mutually cancel in the transverse plane. This collective effect gives rise to a power suppressed scaling [37]

$$
\Sigma\left(p_{\perp}\right) \sim \mathcal{O}\left(p_{\perp}^{2}\right) .
$$

As $p_{\perp}$ increases, but still remains small compared to $m_{H}$, the distribution is described by kinematic configurations with $p_{\perp} \sim k_{\perp i} \ll m_{H}$. As discussed in appendix A.1, in this region the cumulative distribution features an exponential suppression of the form

$$
\Sigma\left(p_{\perp}\right) \sim \sigma_{0} \exp \left\{-\bar{\alpha} \ln ^{2} \frac{m_{H}}{p_{\perp}}\right\}
$$

where $\bar{\alpha}=2 C_{A} \alpha_{s} / \pi$.

\footnotetext{
${ }^{3}$ This is valid at all logarithmic orders.

${ }^{4}$ Clearly, all integrals in eq. (2.5) are divergent in the soft and collinear limits, and require regularization. However, the final result eq. (2.5) does not depend on the regularization procedure.
} 
At larger transverse momenta $\left(p_{\perp} \sim m_{H}\right)$ the approximation that led to eq. (2.5) is not justified anymore. Therefore, in this region one has to smoothly switch from the resummed prediction to the fixed-order one, where the effect of the hard radiation is treated correctly. This can be done for example using the following matching formula

$$
\Sigma\left(p_{\perp}\right)=\Sigma^{\text {resum }}\left(p_{\perp}\right)+\left(\Sigma^{\text {f.o. }}\left(p_{\perp}\right)-\mathcal{T}^{\text {f.o. }}\left[\Sigma^{\text {resum }}\left(p_{\perp}\right)\right]\right),
$$

where we indicate with $\mathcal{T}^{\text {f.o. }}[f]$ the fixed-order expansion of $f$. At small $p_{\perp}$ the difference between the fixed-order result and the Taylor expansion of the resummed result is free of logarithmically-enhanced terms

$$
\lim _{p_{\perp} \rightarrow 0}\left(\Sigma^{\text {f.o. }}\left(p_{\perp}\right)-\mathcal{T}^{\text {f.o. }}\left[\Sigma^{\text {resum }}\left(p_{\perp}\right)\right]\right)=\text { const. }
$$

This allows one to extend the fixed-order description to $p_{\perp} \rightarrow 0$ and, at the same time, ensures that all terms that contain large logarithms at low $p_{\perp}$ are resummed.

The precise way to switch from the resummation to the fixed-order description is ambiguous. One source of ambiguity comes from choosing a particular form for the matched cross section (in our example, eq. (2.8), we chose to combine the resummed and fixed-order predictions additively). A second source of ambiguity is connected with the scale at which the transition from resummed to fixed-order result takes place. Although all of these effects are formally of higher-order both in the resummation and fixed-order counting, their numerical impact can be non-negligible. We consider the latter issue in what follows, while leaving a discussion of the choice of the matching scheme to the next section.

In order to switch off resummation effects at large $p_{\perp}$, one can modify the resummed cross section by including controlled power-suppressed corrections. One possible way to do this is to modify the resummed logarithms in eq. (2.7) as follows ${ }^{5}$

$$
L \equiv \ln \frac{m_{H}}{p_{\perp}}=\ln \frac{m_{H}}{Q}+\ln \frac{Q}{p_{\perp}},
$$

where $Q$ is an arbitrary scale of order $m_{H}$. Moreover, we write

$$
\ln \frac{Q}{p_{\perp}} \rightarrow \frac{1}{p} \ln \left(\left(\frac{Q}{p_{\perp}}\right)^{p}+1\right) \equiv \tilde{L}
$$

where $p$ is a positive number. The motivation for the transformations described above is as follows:

- First, we split the resummed logarithm $L$ into the sum of a small logarithm $\ln \left(m_{H} / Q\right)$ (with $Q \sim m_{H}$ ) and a large logarithm $\ln \left(Q / p_{\perp}\right)$. This operation allows us to introduce a generic scale $Q$ which then appears in the resummed result. We can now expand $L$ around $\ln \left(Q / p_{\perp}\right)$, retaining all terms with the desired logarithmic accuracy. Effectively, this implies that $\ln \left(m_{H} / Q\right)$ is treated perturbatively at fixed order.

\footnotetext{
${ }^{5} \mathrm{~A}$ more correct prescription is to modify the logarithms $\ln \left(m_{H} / k_{\perp 1}\right)$ where $k_{\perp 1}$ is the transverse momentum of the hardest emitted gluon. This technicality is avoided here for the sake of clarity, and it will be discussed in appendix A.
} 
In our LL example, for $p_{\perp} \sim k_{\perp i} \ll m_{H}$, this means

$$
\begin{aligned}
\Sigma^{\operatorname{resum}}\left(p_{\perp}\right) \sim e^{-\bar{\alpha} L^{2}} & =\exp \left\{-\bar{\alpha}\left[\ln ^{2} \frac{Q}{p_{\perp}}+2 \ln \frac{Q}{p_{\perp}} \ln \frac{m_{H}}{Q}+\ln ^{2} \frac{m_{H}}{Q}\right]\right\} \\
& =\exp \left\{-\bar{\alpha} \ln ^{2} \frac{Q}{p_{\perp}}+\mathcal{O}\left(\alpha_{s} L\right)\right\}\left(1-\bar{\alpha} \ln ^{2} \frac{m_{H}}{Q}+\mathcal{O}\left(\alpha_{s}^{2}\right)\right) \\
& \simeq \exp \left\{-\bar{\alpha} \ln ^{2} \frac{Q}{p_{\perp}}\right\}
\end{aligned}
$$

where all terms beyond LL were neglected. This prescription is convenient because the $Q$-dependence is always of higher-logarithmic order and, therefore, a $Q$-variation probes the size of subleading logarithms that are not considered in the resummation.

- Second, we modify the logarithm $\ln \left(Q / p_{\perp}\right)$ by including power-suppressed terms that force $\tilde{L}$ to vanish at large $p_{\perp}$. These modifications do not affect the small- $p_{\perp}$ limit. Indeed, it follows from eq. (2.11) that

$$
\tilde{L} \sim \ln \frac{Q}{p_{\perp}}, \text { for } p_{\perp} \ll Q ; \quad \tilde{L} \sim \frac{1}{p}\left(\frac{Q}{p_{\perp}}\right)^{p}, \text { for } p_{\perp} \gg Q .
$$

As a consequence, the resummation scale $Q$ and the scaling parameter $p$ must be chosen in such a way that the high- $p_{\perp}$ scaling of the resummed component (and its fixed-order expansion) does not modify the scaling of the fixed-order prediction. This means that $p$ and $Q$ are to be chosen in such a way that the resummed component vanishes more quickly than the fixed-order result for $p_{\perp} \gtrsim Q$.

The above discussion shows that $Q$ is indeed the scale at which the transition between resummed and fixed-order results occurs. Similarly to the renormalization and factorization scales, its choice is ambiguous, although certain conditions should be satisfied. Indeed, it is clear that (a) $Q$ should not be too different from $m_{H}$, to ensure that $\ln Q / m_{H}$ are not large and (b) it should approximately correspond to the scale at which the soft and collinear approximations to the matrix element and kinematics break down. In practice, one can choose $Q$ by comparing the exact result $\Sigma^{\text {f.o. }}$ with the expansion of the resummed result $\mathcal{T}^{\text {f.o. }}\left[\Sigma^{\text {resum }}\right]$, and set $Q$ to the $p_{\perp}$ scale at which the two start to significantly deviate from each other. This is illustrated in figure 1, which shows the difference between the LO differential $p_{\perp}$ spectrum and the expansion of the resummed result at the same order. Specifically, we plot

$$
\left|\left(\frac{d \sigma^{\mathrm{LO}}}{d p_{\perp}}-\mathcal{T}^{\mathrm{LO}}\left[\frac{d \sigma^{\mathrm{resum}}}{d p_{\perp}}\right]\right) / \frac{d \sigma^{\mathrm{LO}}}{d p_{\perp}}\right| .
$$

We observe that when only the top contribution is included (solid, red curve), the logarithmic terms account for about half of the fixed-order result at scales $p_{\perp} \sim 50-60 \mathrm{GeV}$. This suggests that the resummation scale should be of this order. We conventionally choose $Q=m_{H} / 2$ as a central value. As far as the choice of the parameter $p$ is concerned, we have to ensure that at large $p_{\perp}$ the resummed component vanishes faster than the fixed order. Considering the asymptotic scaling in eq. (2.13), we choose $p=4$ which guarantees that the differential distribution vanishes as fast as $1 / p_{\perp}^{5}$ for $p_{\perp} \gtrsim Q$. In principle, any value 


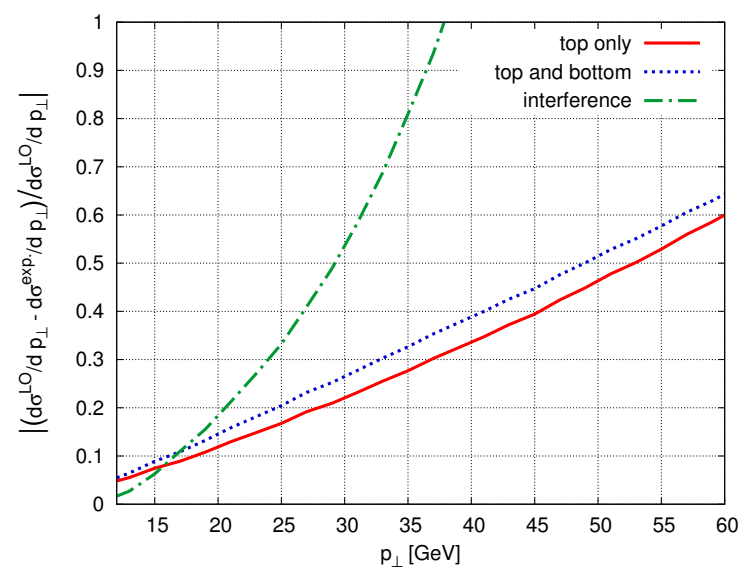

Figure 1. Differences between leading-order distributions and their logarithmic contributions, normalized to the leading-order results. The three curves show the case for top quark (solid/red), top and bottom quarks (dotted/blue), and top-bottom interference (dot-dashed/green). See text for details.

of $p$ greater than 3 will equally do, since $p$ only determines how fast the resummation is turned off above the scale $Q$. We have indeed checked that by varying $p$ by one unit around $p=4$ the results do not change significantly.

The same figure shows results for the full spectrum where both top and bottom loops are included (dotted, blue curve), and results for top-bottom interference (dot-dashed, green curve). ${ }^{6}$ In this case the situation changes considerably, and this will be the subject of the next section.

\section{$2.2 \quad$ Issues with $b$ quarks}

The "standard" approach to resummation described in section 2.1 becomes problematic in case of the Higgs boson production in hadron collisions [34, 35, 38-41]. The difficulty is related to the fact that the $g g H$ vertex is not point-like but, rather, is induced by a quark loop. The presence of the quark loop implies the existence of structure-dependent radiation with peculiar properties and has important consequences for the resummation. The key to the following discussion is the appreciation of the fact that the structure-dependent radiation is suppressed if $p_{\perp}$ is smaller than the mass of the quark but it becomes important otherwise. For $p_{\perp}$ larger than the quark mass, the soft and collinear approximations that provide the foundation for small $p_{\perp}$ resummation become unreliable, as they focus on emissions off external lines and systematically neglect structure-dependent effects. In this section we elaborate on this issue.

We consider Higgs boson production in gluon fusion mediated by a quark loop. We denote the mass of the quark by $m_{q}$ and consider two cases $m_{q} \gg m_{H}$ and $m_{q} \ll m_{H}$. In the first case, the structure dependence enters at $p_{\perp} \gtrsim m_{q} \gg m_{H}$, so that emissions off the external lines dominate for transverse momenta up to the Higgs mass and even higher. Therefore, if we restrict ourselves to values of $p_{\perp}$ that are comparable to $m_{H}$, the situation

\footnotetext{
${ }^{6}$ We ignore the bottom squared contribution, which is completely negligible in the Standard Model.
} 
is no different from point-like interaction, and there are no issues in the resummation procedure described in the previous section. In the Standard Model, this is indeed what happens with the top loop contribution to the Higgs boson transverse momentum spectrum.

The second case, $m_{q} \ll m_{H}$ is very different. Indeed, in this case there are three distinct regions $p_{\perp} \lesssim m_{q}, m_{q} \lesssim p_{\perp} \lesssim m_{H}$ and $m_{H} \lesssim p_{\perp}$. In the first region $p_{\perp} \lesssim m_{q}$, the transverse momenta of the Higgs boson and the recoiling partons are typically small enough not to resolve the structure of the loop and the extra radiation factorizes. For the bottom-quark contribution $\left(m_{q} \sim 5 \mathrm{GeV}\right)$ the effect of additional QCD radiation is strongly suppressed in this region by all-order effects, so that its impact on the total cross section is small. ${ }^{7}$ Note, however, that in this region there are large logarithmic contributions of the type $\ln ^{2} m_{H} / m_{b}$, whose resummation is not fully understood even at the lowest perturbative order [42, 43].

In the second region $m_{q} \lesssim p_{\perp} \lesssim m_{H}$ the structure-dependent radiation becomes essential and the $g g H$ vertex does not factorize. In addition to the usual logarithms $\ln m_{H} / p_{\perp}$, the radiation gives rise to logarithms $\ln p_{\perp} / m_{q}$ and $\ln m_{H} / m_{q}$, whose origin and potential resummation are not well understood. ${ }^{8}$

The reason why the small- $p_{\perp}$ resummation is problematic in this region is the following. Emissions off internal lines can become as important as emissions off external lines, and together they probe the loop structure of the $g g H$ vertex. It follows that approximating the small $p_{\perp}$ region with an on-shell $g g H$ form factor is not justified. In particular, while form factor effects in the top-quark case only introduce $\left(p_{\perp} / m_{H}\right)$-suppressed corrections, in this case they both introduce a new logarithmic structure $\left(\ln p_{\perp} / m_{q}\right.$ and $\left.\ln m_{H} / m_{q}\right)$ and suppress radiation with $p_{\perp} \gtrsim m_{q}$. In other words, while in the top case, described in section 2.1, at finite $p_{\perp}$, the coefficients of the logarithms differ from the resummed result by $p_{\perp}$-suppressed terms, in the $b$-quark case, this difference contains new logarithmic terms $\ln p_{\perp} / m_{b}$ and $\ln m_{H} / m_{b}$ in the region $m_{b} \lesssim p_{\perp} \lesssim m_{H}$.

As a consequence, the collinear approximation should not be expected to work far away from the $b$-quark threshold. To quantify this effect, we go back to figure 1 . We see that, while for the top-only case (solid, red line) the collinear approximation to the leading order accounts for half of the result at about $p_{\perp} \sim 50-60 \mathrm{GeV}$, for the top-bottom interference this scale is reduced to about $30 \mathrm{GeV}$. When top and bottom contributions are considered together, this effect is less dramatic since in the SM the interference accounts for about $\sim 5 \%$ of the full result. This can be seen in the dotted blue curve of figure 1 .

Because of the above issues, it is clear that constructing a reasonable description of the $b$-quark contribution to the Higgs transverse momentum distribution is problematic. Since, as we already stressed, in this case the resummation of potentially large logarithms is not entirely understood, the best we can do is to use different ways to interpolate between regions of small and large transverse momenta and check to what extent the different results are compatible.

As already stated, the Higgs boson production in the Standard Model is dominated by the top quark loop; the bottom loop provides a very small contribution that is lifted up to

\footnotetext{
${ }^{7}$ This region is also very sensitive to non-perturbative effects.

${ }^{8}$ For some recent studies, see e.g. refs. [42-44].
} 
$\mathcal{O}(-5 \%)$ by its interference with the top amplitude. Because of this, a $\mathcal{O}(20-30 \%)$ control on the top-bottom interference is sufficient to control the Higgs transverse momentum spectrum at the few percent level. With this in mind, we now study in more detail the different ways to treat the bottom contribution.

One option is to apply eq. (2.8) with the resummation scale set to $Q \sim m_{b}$ [34]. This choice is equivalent to employing fixed-order description for all values of transverse momenta. Indeed, for $1 \mathrm{GeV} \lesssim p_{\perp} \lesssim Q \sim m_{b}$, the resummed logarithms $\ln \left(Q / p_{\perp}\right)$ never become large and for $p_{\perp} \gtrsim Q$ the fixed-order result is adopted anyhow. Since a typical error made within this approach is provided by uncalculated higher order terms, if we use a NLO computation for the interference, we make an error of $\operatorname{order}^{9}\left[\alpha_{s} /(2 \pi)\right]^{2} \ln ^{4}\left(m_{H} / m_{b}\right)$ and $\left[\alpha_{s} /(2 \pi)\right]^{2} \ln ^{4}\left(p_{\perp} / m_{H}\right)$ which both evaluate to $15-20$ percent, for $p_{\perp} \sim m_{b} \sim 5 \mathrm{GeV}$.

The previous option amounts to neglecting the resummation for the top-bottom interference and to using the fixed-order result for all transverse momenta; the other extreme alternative consists of extending the resummation beyond its established domain of validity. We can do this by using the same resummation scale, $Q \sim m_{H} / 2$, both for the top and the top-bottom interference contributions $[35,38]$. In this case, at low $p_{\perp}$ we introduce $\operatorname{logarithms} \ln p_{\perp} / Q$ in the interference through the resummation prescription which are not guaranteed to be correct and, by doing that, we effectively introduce errors that are similar to those discussed above. At higher $p_{\perp}$ the impact of these logarithms becomes smaller since at $p_{\perp} \sim Q$ the resummation effects smoothly turn off and we recover the fixed order prediction. Hence, when we under- or over-resum logarithms we expect comparable $\mathcal{O}(20 \%)$ theoretical errors on the interference contribution to the Higgs $p_{\perp}$ spectrum. An important question is whether these different sources of uncertainties pull the predictions apart or they remain compatible with each other.

Before concluding this section, we mention that within the additive matching scheme of eq. (2.8) the resummation term, which is proportional to the lowest-order form factor at zero transverse momentum, is added to the fixed order result. As we mentioned earlier, the form factor effects lead to a dependence of the leading order amplitude on $p_{\perp}$, that is not captured in this approach. To account for this, we also consider a multiplicative matching scheme, which can be schematically defined as ${ }^{10}$

$$
\Sigma\left(p_{\perp}\right)=\Sigma^{\text {resum }}\left(p_{\perp}\right) \mathcal{T}^{\text {f.o. }}\left[\frac{\Sigma^{\text {f.o. }}\left(p_{\perp}\right)}{\Sigma^{\text {resum }}\left(p_{\perp}\right)}\right]
$$

Similarly to eq. (2.8), eq. (2.15) smoothly interpolates between a low $p_{\perp} \ll Q$ region, where resummation dominates, to a large $p_{\perp} \gg Q$ region, where the result is obtained from a fixed order calculation. Clearly, the fixed order accuracy is preserved in the $p_{\perp} \rightarrow 0$ limit. The main difference with eq. (2.8) is that now the higher order terms induced by the resummation in the transition region are weighted with the fixed (lower) order result at finite transverse momentum. This should at least partially capture the $p_{\perp}$ dependence of the exact higher order amplitude, and lead to a more realistic description of the physics. Because

\footnotetext{
${ }^{9}$ Note that these estimates refer to the top-bottom interference contribution. As we said, the term proportional to $y_{b}^{2}$ is negligible in the Standard Model.

${ }^{10}$ The actual implementation of this procedure requires extra care, as described in appendix A.3.
} 
of this, we choose the multiplicative matching scheme eq. (2.15) as our default matching scheme. Nevertheless, matching ambiguities are by construction of higher-order nature and, therefore, any matching prescription is formally equally valid. Differences between matching prescriptions can be used to estimate the uncertainty in the transition region.

\section{Results}

\subsection{Inclusion of bottom-quark loops and matching uncertainties}

In this section we describe the practical and technical implementation of the top-bottom interference in the resummation and matching, and the uncertainty associated with it. As we described in section 2.2, the rigorous resummation in the presence of the bottom-quark loop is currently impossible. To remedy this problem, we adopt different approaches to include this contribution in the matched result. We use the arbitrariness in the choice of the resummation scale associated with the top-bottom interference and in the choice of the matching scheme to assess the inherent ambiguity of the resummation procedures.

We start by discussing the resummation scale. We treat separately the contribution of the top-squared amplitude and the top-bottom interference. ${ }^{11}$ In particular, we associate two different resummation scales with the top and the interference contributions, and we use the following notation to denote the various cumulative distributions

$$
\begin{aligned}
\Sigma_{t+b}\left(p_{\perp}, Q_{t}, Q_{b}\right) & \rightarrow \text { top and bottom, including the interference; } \\
\Sigma_{t}\left(p_{\perp}, Q_{t}\right) & \rightarrow \text { only top. }
\end{aligned}
$$

As explained in section 2.1, for the top-only contribution we set the resummation scale to $Q_{t}=m_{H} / 2$. For the interference, instead, we use the following prescriptions to quantify the associated uncertainty (see section 2.2):

- We choose $Q_{b} \sim m_{b}$, effectively switching off the resummation for the interference at scales of the order of the bottom mass. As it was initially suggested in ref. [34], we choose $Q_{b}=2 m_{b}$ as our central scale. This is achieved by computing

$$
\Sigma_{t+b}\left(p_{\perp}, m_{H} / 2,2 m_{b}\right)=\Sigma_{t}\left(p_{\perp}, m_{H} / 2\right)+\Sigma_{t+b}\left(p_{\perp}, 2 m_{b}, 2 m_{b}\right)-\Sigma_{t}\left(p_{\perp}, 2 m_{b}\right) .
$$

This implies that in the region of transverse momenta that we are interested in, the interference is described only at fixed order and no resummation for this contribution is performed.

- We consider the opposite situation in which we rely on the collinear approximation also for $m_{b} \ll p_{\perp}$, and simply treat the new logarithmic terms that appear above this scale as a regular remainder that can be described at fixed order. As a consequence, the resummation for the interference contribution is switched off, as in the top-only case, at scales of order $60 \mathrm{GeV}$. We choose $Q_{t}=Q_{b}=m_{H} / 2$ as our central scale, for simplicity.

\footnotetext{
${ }^{11}$ For our numerical results, we also include the bottom squared contribution, which is however negligible in the Standard Model.
} 
In both approaches, logarithms of the ratio $p_{\perp} / m_{b}$ are not resummed. Although in the region $m_{b} \ll p_{\perp} \ll m_{H}$ these logarithmic terms can be potentially large and therefore should be included to all orders, recent studies seem to suggest that an accurate prediction of these terms is achieved by considering the first few terms in the fixed-order perturbative expansion $[42,43]$.

As far as the resummation is concerned, the result will be nearly identical to the $m_{q} \rightarrow \infty$ one. ${ }^{12}$ The only difference is that now the Born squared amplitude and the hard-virtual correction will contain the full dependence on the top and bottom masses. In particular, no modification of the $p_{\perp}$-dependent radiation pattern is introduced. Technically, we implement the LO and the NLO amplitudes for $g g \rightarrow H$ with full mass dependence following ref. [45].

We now study numerically the difference between the two prescriptions for the bottom resummation scale. We start by introducing the setup that we adopt for our predictions. We consider proton collisions at the $13 \mathrm{TeV}$ LHC. The Higgs boson mass is taken to be $m_{H}=125 \mathrm{GeV}$ and the top and bottom pole masses ${ }^{13}$ are set to $m_{t}=173.2 \mathrm{GeV}$ and $m_{b}=4.75 \mathrm{GeV}$, respectively. We work within a fixed flavor-number scheme $\left(n_{F}=5\right)$ and use the PDF4LHC15_nnlo set of parton distribution functions [46] interfaced through LHAPDF6 [47]. We use the value of the strong coupling constant $\alpha_{s}$ provided by the PDF set. As central values for the renormalization and factorization scales we take

$$
\mu_{R}=\mu_{F}=M_{T} / 2, \quad \text { with } \quad M_{T}=\sqrt{m_{H}^{2}+p_{\perp}^{2}} .
$$

In order to estimate the perturbative uncertainties for each prediction, we perform a 7-point variation of the factorization $\left(\mu_{F}\right)$ and renormalization $\left(\mu_{R}\right)$ scales around the central value by a factor of two. Moreover, we vary $Q_{t}$ and $Q_{b}$ by a factor of two around their respective central values, keeping fixed $\mu_{R}=\mu_{F}=M_{T} / 2$. The final uncertainty band is obtained as the envelope of all above variations. As a default, we adopt the multiplicative scheme discussed in section 2.2 and described in detail in appendix A.3.

The fixed-order NLO results for the top-bottom interference are based on the calculation presented in [36], which in turn comprises the two-loop amplitudes for $g g \rightarrow H g$, $q \bar{q} \rightarrow H g$ and $q g \rightarrow H q$ derived in [32,33] together with corresponding loop-squared real radiation amplitudes as provided by OpenLoops [48, 49] combined with Collier [50]. For the Monte Carlo integration and subtraction the PowHEG-Box-RES is used [51, 52].

We now discuss the dependence on the choice of the resummation scale associated with the bottom contribution. We start by comparing results for the top-bottom interference for two values of the resummation scale $Q_{b}$. The results are displayed in the left plot in figure 2. The two predictions differ by about $20 \%$ for $p_{\perp} \sim 30 \mathrm{GeV}$, in line with what we expected from the discussion in section 2.2. We note, however, that although the two results are computed for very different choices of the resummation scales, they are still compatible (although marginally for $p_{\perp} \gtrsim 25 \mathrm{GeV}$ ) within their respective uncertainties. Only for $p_{\perp} \gtrsim 50 \mathrm{GeV}$ the two results differ significantly, since the interference obtained

\footnotetext{
${ }^{12} \mathrm{HEFT}$ in the following.

${ }^{13}$ We work in the on-shell renormalization scheme as a default.
} 

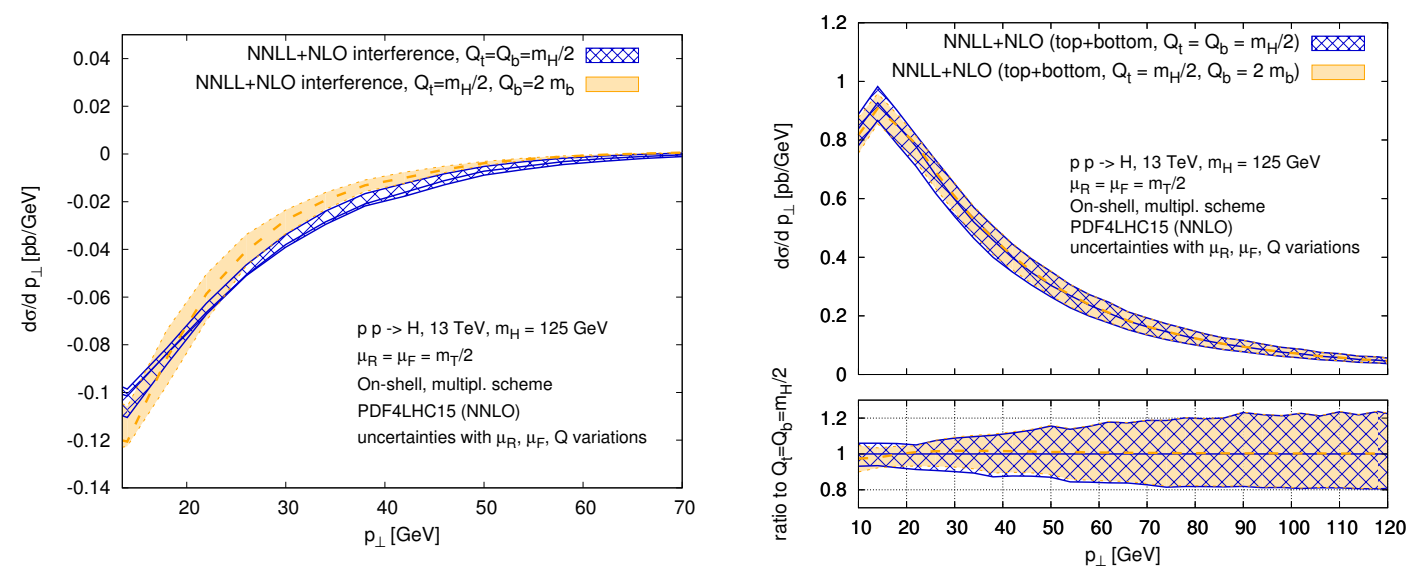

Figure 2. Comparison between two resummation-scale prescriptions for top-bottom interference (left) and full (right) distributions. See text for details.

with $Q_{b}=2 m_{b}$ vanishes faster than the one obtained with $Q_{b}=m_{H} / 2$. However, in this region the contribution of the interference to the physical spectrum is completely negligible. Each of the two results has a relative uncertainty of about $15 \%$ for $p_{\perp} \lesssim 40 \mathrm{GeV}$. The variations of the resummation scales around their central value, and the variation of $\mu_{R}$ and $\mu_{F}$ have a similar impact on the final band.

The right plot of figure 2 shows an analogous comparison for the transverse momentum distribution that includes both top and bottom contributions. Since the interference only accounts for about $5 \%$ of the full result, we find that the two resummation prescriptions for the top-bottom interference are indistinguishable within the uncertainties of the top contribution. Indeed, in this case the uncertainty band is dominated by the $\mu_{R}$ and $\mu_{F}$ variation of the top contribution, which amounts to about $10-15 \%$ for $p_{\perp} \lesssim 40 \mathrm{GeV}$, while the resummation-scale uncertainty amounts to about $5 \%$ in this region. Note however that the top-only contribution has been computed one order higher, both in fixed-order QCD [7-11] and in the resummation framework [29]. In this paper, we focus on the $b$-quark effects and hence do not include these results but, as a matter of principle, they can be used to further reduce the uncertainty on the top contribution.

We now investigate the second source of resummation ambiguity, namely the choice of the matching scheme. As discussed in section 2.2, besides our default multiplicative scheme we also consider an additive scheme. Both schemes are precisely defined in appendix A.3. We remind the reader that, as far as the top-bottom interference is concerned, the main qualitative difference between the two approaches is that within the additive matching scheme, the resummation contribution is proportional to the $g g \rightarrow H$ form factor at zero transverse momentum whereas in the multiplicative matching scheme it is weighted by the form factor $g^{*} g^{*} \rightarrow H$ at finite transverse momentum. In order to study this source of ambiguity more precisely, we consider the additive matching scheme, with two different scales $\left(Q_{b}=m_{H} / 2\right.$ and $\left.Q_{b}=2 m_{b}\right)$, and compare the results to the multiplicative scheme.

Since in the additive scheme the resummed contribution does not include form-factor effects, we expect sizable differences between results obtained with $Q_{b}=m_{H} / 2$ and 

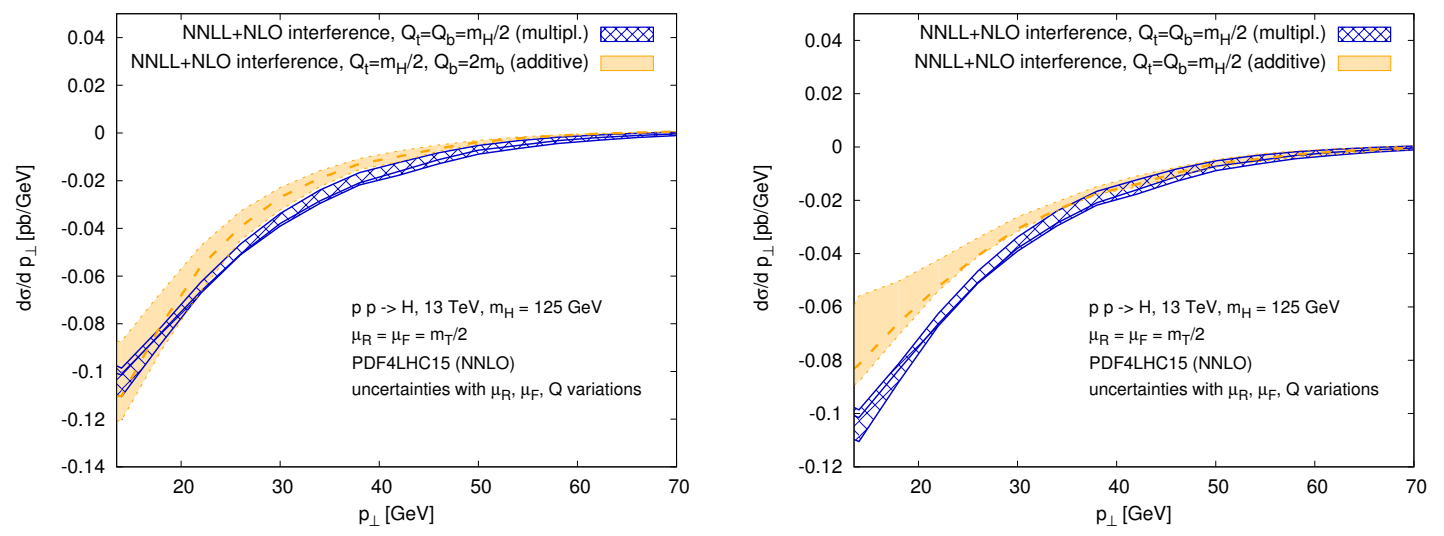

Figure 3. Comparison between the additive scheme with $Q_{t}=m_{H} / 2, Q_{b}=2 m_{b}$ (left plot) or $Q_{t}=Q_{b}=m_{H} / 2$ (right plot), and the default multiplicative scheme with $Q_{t}=Q_{b}=m_{H} / 2$.

$Q_{b}=2 m_{b}$. We recall that this is not the case in the multiplicative scheme (see figure 2) where form-factors effects are automatically accounted for. We then show, in figure 3 , the comparison between the top-bottom interference in the default multiplicative scheme with $Q_{b}=m_{H} / 2$ and the additive scheme with $Q_{b}=2 m_{b}$ (left plot) and $Q_{b}=m_{H} / 2$ (right plot). We observe that the difference between the two schemes is larger when the additive scheme with $Q_{b}=m_{H} / 2$ is used. Nevertheless, we find that also in this case, the difference between the two schemes for the interference does not exceed $\sim 20 \%$ in the bulk of the distribution. Again, the full transverse momentum distribution, shown in the left plot of figure 4 , is only mildly affected by this ambiguity.

Finally, in the right plot of figure 4, we show the ratio of the full distribution computed using the default multiplicative scheme, to the corresponding HEFT result. The default result, i.e. multiplicative matching scheme with $Q_{t}=Q_{b}=m_{H} / 2$, is in good agreement with the NLO prediction. For comparison, we also report the other extreme solution obtained with the multiplicative scheme with $Q_{t}=m_{H} / 2, Q_{b}=2 m_{b}$. We observe that this choice is in good agreement with both the fixed order and the default matched solution.

In summary, we find that a conservative approach towards the inclusion of bottom-mass effects in the matched prediction for the Higgs- $p_{\perp}$ spectrum leads to a $\sim 20 \%$ ambiguity on the top-bottom interference in the region $m_{b} \lesssim p_{\perp}$. Since the interference provides a rather small contribution to the Higgs transverse momentum distribution, this ambiguity translates into a few-percent uncertainty in the Higgs $p_{\perp}$ spectrum.

In what follows, we will use the result obtained with the multiplicative matching scheme with $Q_{t}=Q_{b}=m_{H} / 2$ as our central value. To estimate uncertainties, we will consider the envelope of scale variations and the result obtained either by using the multiplicative or the additive scheme with $Q_{t}=m_{H} / 2, Q_{b}=2 m_{b}$. In addition to these source of uncertainty, an additional ambiguity arises from the choice of the renormalization scheme for the quark masses. This will be discussed in the next section. 

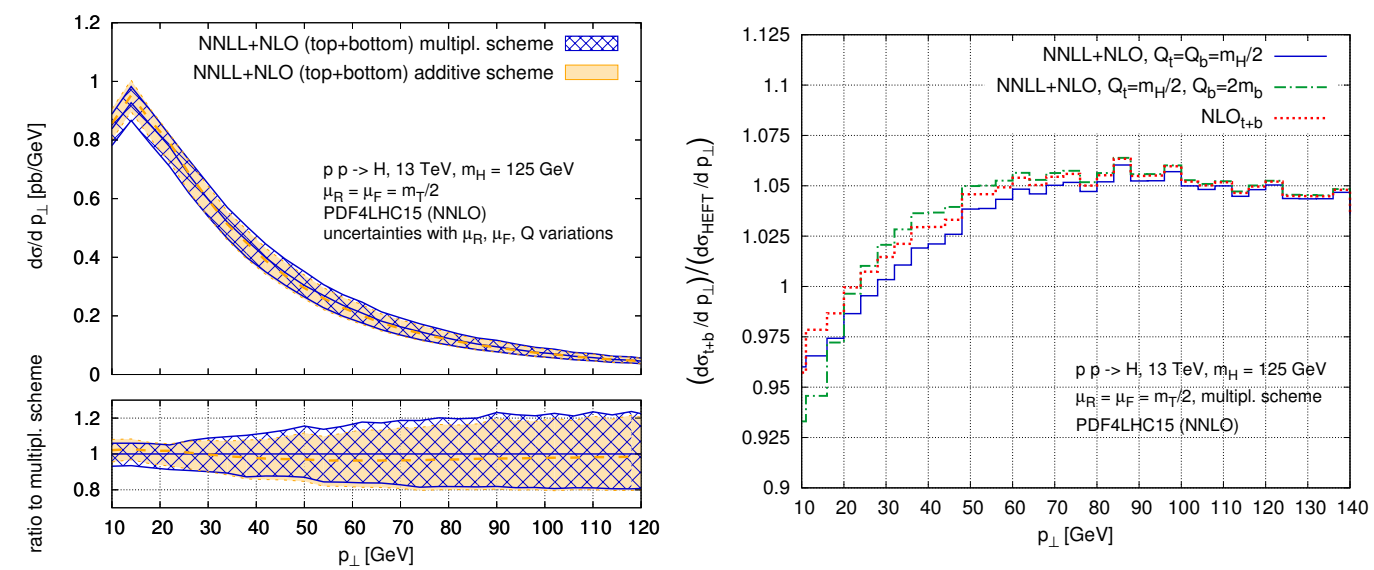

Figure 4. Left plot: comparison for the full distribution between the additive scheme with $Q_{t}=$ $Q_{b}=m_{H} / 2$, and the default multiplicative scheme with $Q_{t}=Q_{b}=m_{H} / 2$. Right plot: ratio of the full distribution with different accuracies to the HEFT prediction based on the same accuracy. In the right plot all matched results are obtained within the multiplicative matching scheme.
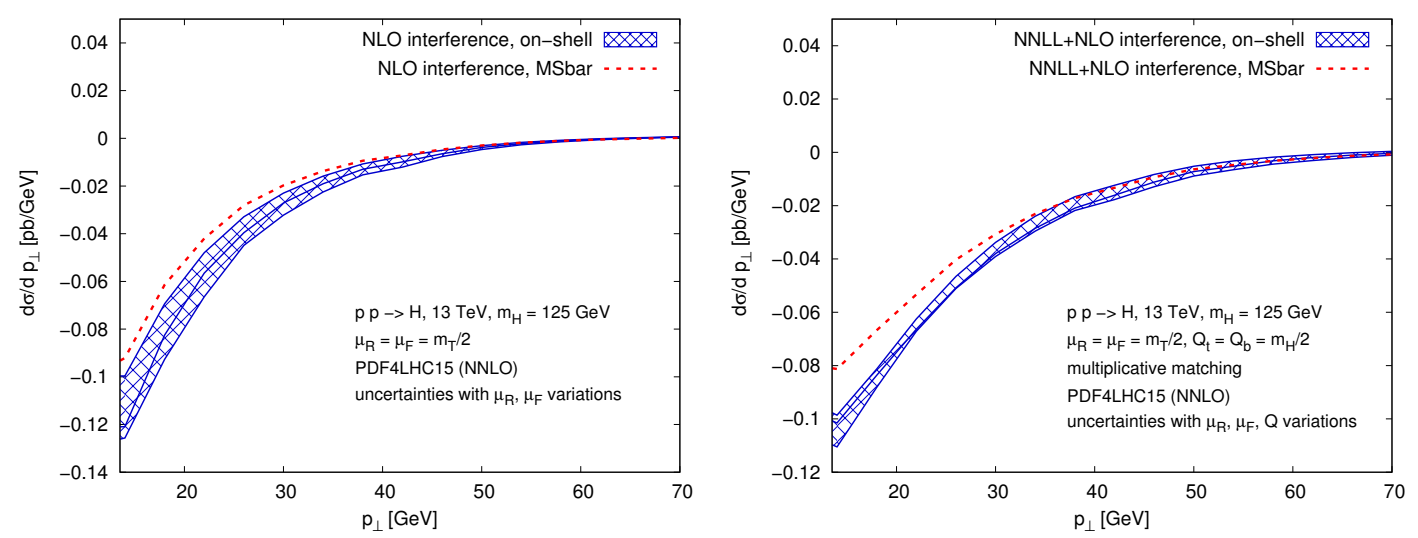

Figure 5. The top-bottom interference contribution for the fixed order (left) and the matched (right) distributions, for different choices of the mass-renormalization scheme. See text for details.

\subsection{Mass-scheme uncertainty and final results}

In this section, we present our final results for the NNLL+NLO matched distributions. We use as default the multiplicative matching scheme with resummation scales $Q_{t}=Q_{b}=m_{H} / 2$. We renormalize the bottom-quark mass in the on-shell scheme. To estimate the uncertainty we change the details of the resummation and matching as explained in the previous section. In addition, we consider a different renormalization scheme for the bottom quark mass to estimate the related uncertainty. To this end, we employ the $\overline{\mathrm{MS}}$ renormalization scheme. We take the mass renormalization scale to be $100 \mathrm{GeV}$, and use $m_{b}=m_{b}^{\overline{\mathrm{MS}}}(100 \mathrm{GeV})=3.07 \mathrm{GeV}$ as an input parameter. ${ }^{14}$

In figure 5 we display the results for the top-bottom interference contribution. The fixed order result is presented in the left plot. We show the uncertainty band for the on-shell

\footnotetext{
${ }^{14} \mathrm{We}$ calculated this value using the program RunDec $[53,54]$ with the input value $m_{b}^{\overline{\mathrm{MS}}}\left(m_{b}^{\overline{\mathrm{MS}}}\right)=4.2 \mathrm{GeV}$.
} 

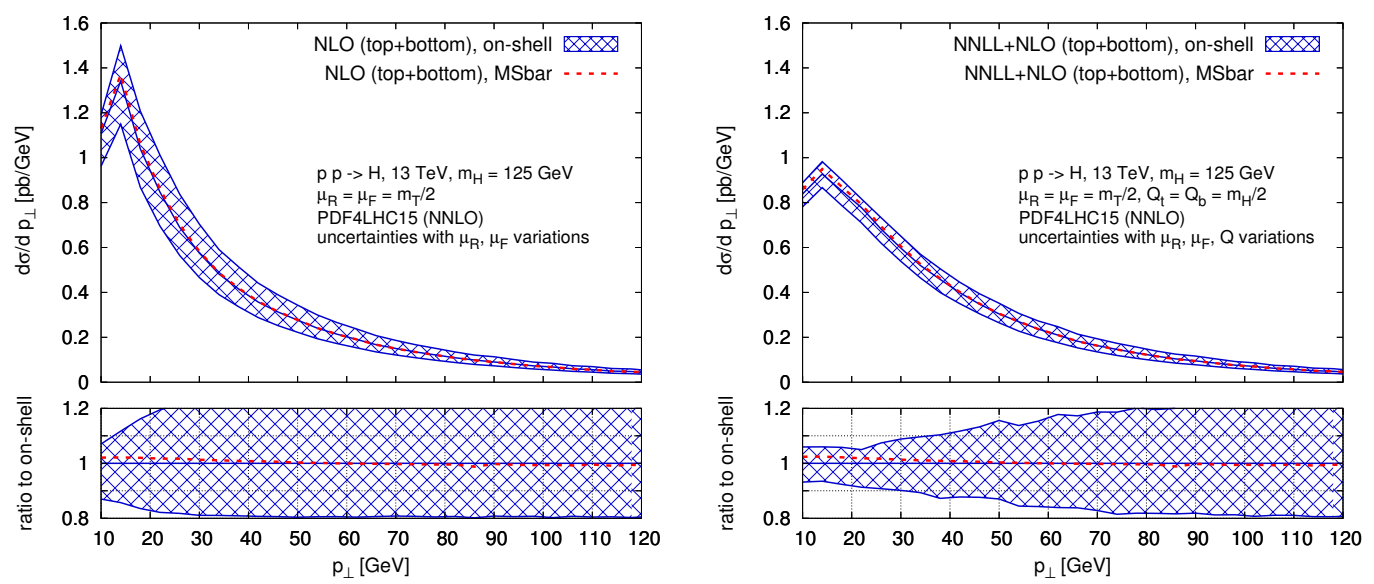

Figure 6. The full top plus bottom distribution for the fixed order (left) and the matched (right) results, for different choices of the mass renormalization scheme. See text for details.

mass-renormalization scheme and the central value for the $\overline{\mathrm{MS}}$ scheme. The uncertainty band is calculated from a 7-point scale variation. We see that the scheme ambiguity is larger than the scale variation, as already observed in ref. [36]. The right plot shows our results for the matched distributions with the two different mass schemes. The difference between the two bottom-mass schemes is similar to the fixed order case, but since now the matched prediction has a smaller uncertainty, the separation between the two results is more significant. As follows from figure 5, the top-bottom interference contribution has an ambiguity of about $15-20 \%$ down to $p_{\perp} \sim 10 \mathrm{GeV}$. In order to improve on this, one would need a NNLO calculation for the top-bottom interference, which is currently out of reach.

The analogous plots for the full distribution that includes both top and bottom amplitudes are shown in figure 6, for the fixed order (left) and resummed (right) results. Unlike for the top-bottom interference contribution, in this case the difference between the two results for the bottom-mass schemes are much smaller, at the level of a few percent. This is because the top-bottom interference contributes to just $\mathcal{O}(-5 \%)$ of the full spectrum. ${ }^{15}$

Our best current predictions for the top-bottom interference and full $p_{\perp}$ spectrum including all the relevant uncertainties are shown in figure 7. As discussed earlier, the uncertainty bands are obtained as an envelope of:

- a 7-point renormalization and factorization scale variation;

- resummation scale variation $Q_{t}=Q_{b} \in\left\{m_{H} / 4, m_{H} / 2, m_{H}\right\}$ for $\mu_{R}=\mu_{F}=M_{T} / 2$;

- multiplicative matching scheme with $Q_{t}=m_{H} / 2, Q_{b}=2 m_{b}$ for $\mu_{R}=\mu_{F}=M_{T} / 2$;

- additive matching scheme with $Q_{t}=m_{H} / 2, Q_{b}=2 m_{b}$ for $\mu_{R}=\mu_{F}=M_{T} / 2$.

In addition, if the default matched result but in the $\overline{\mathrm{MS}}$ renormalization scheme for the bottom-quark mass is outside the uncertainty band estimated as described above, we extend

\footnotetext{
${ }^{15}$ Although the bottom-mass scheme ambiguity has a very moderate impact on the SM Higgs $p_{\perp}$ spectrum, this effect might be more significant for specific BSM scenarios. A dedicated study of such scenarios is necessary in order to assess the theory uncertainties precisely.
} 

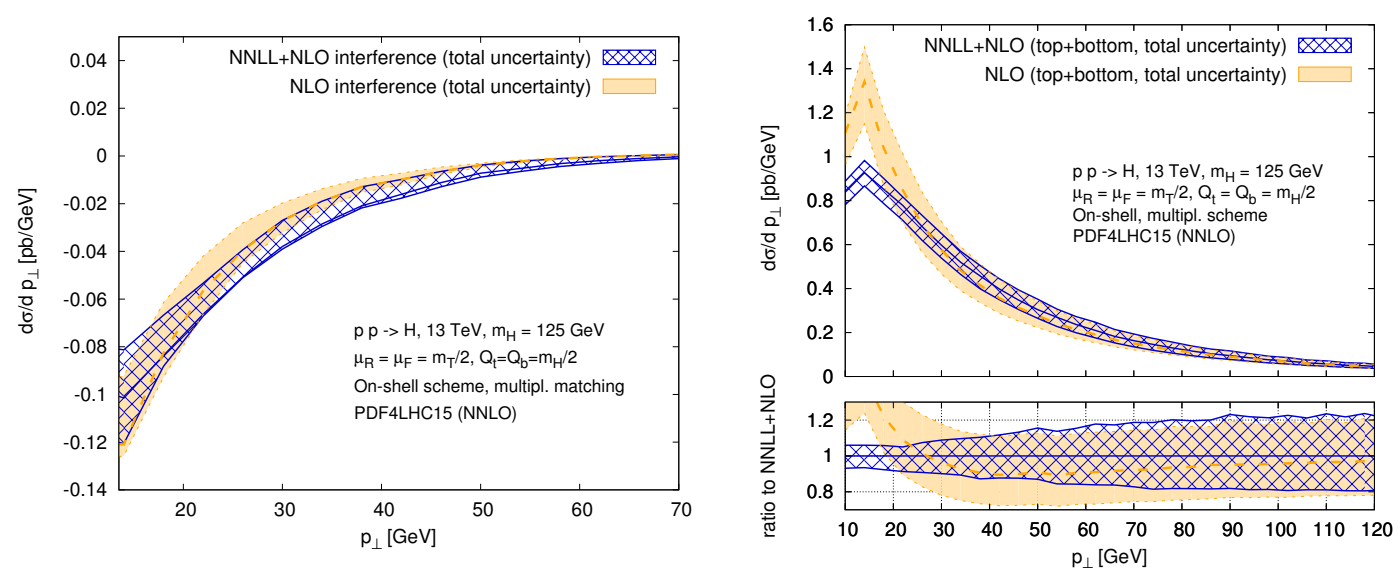

Figure 7. The distributions for the top-bottom interference contribution (left) and the full NNLL matched result (right), using the multiplicative scheme with resummation scale $Q_{b}=Q_{t}=m_{h} / 2$ as central values. See text for details.

the uncertainty band to accommodate the mass scheme ambiguity. In fact, as shown in figure 5, the latter ambiguity is the major source of uncertainty for the top-bottom interference for transverse momenta below $30 \mathrm{GeV}$.

The top-bottom interference is shown in the left plot of figure 7 . The qualitative features of the fixed-order result are unchanged by the resummation, which however has a noticeable effect on the shape of the distribution. Our final result has an uncertainty of about $\sim 20 \%$, and is compatible with the fixed-order one. In the right plot of figure 7 we present the results for the full spectrum. At large values of the Higgs $p_{\perp} \gtrsim 30 \mathrm{GeV}$ the fixed order result is contained in the error band of the resummed result. However at smaller values, $p_{\perp} \lesssim 30 \mathrm{GeV}$ we observe a marked difference between the two results. The error for the full matched result is close to $10 \%$ for $p_{\perp} \lesssim 30 \mathrm{GeV}$ and close to $\sim 20 \%$ at larger $p_{\perp}$. We stress however that the uncertainty on the dominant top contribution can be further reduced by employing the results of refs. [7-11, 29, 55].

\section{Conclusions}

In this paper we performed a detailed study of the Higgs transverse momentum distribution, focusing on the region of intermediate values of transverse momenta, $m_{b} \lesssim p_{\perp} \lesssim m_{H}$. Indeed, a precise theoretical control of the Higgs $p_{\perp}$ distribution in this region is essential to test the Higgs sector of the Standard Model. In particular, it provides a rare opportunity to probe the Yukawa couplings of light quarks, which are currently poorly constrained. In fact, although the main contribution to the Higgs production cross section is due to the coupling of the Higgs to top quarks, the coupling to bottom quarks has a non-negligible impact on the total cross section through its interference with the top, decreasing the cross section by about $\mathcal{O}(5 \%)$.

The theoretical description of the Higgs $p_{\perp}$ distribution for $m_{b} \lesssim p_{\perp} \lesssim m_{H}$ in QCD is particularly challenging since, once the contribution of bottom quarks is included, the perturbative cross section for small $p_{\perp}$ suffers from the presence of potentially large logarithms 
$\ln \left(p_{\perp} / m_{b}\right), \ln \left(m_{H} / m_{b}\right)$, which can spoil the convergence of the perturbative expansion. The physical origin of these large logarithms is not yet fully understood, and their all-order resummation remains currently out of reach.

Given these conceptual limitations, we provided our best theoretical description of the Higgs $p_{\perp}$ distribution at NNLL+NLO QCD for moderate values of the transverse momentum, including dependence on the bottom mass. An important part of our study was a proper assessment of the theory uncertainty of our results. The NLO result for the topbottom interference suffers from scale uncertainties, which amount to around 15\%. On top of this, a non-negligible source of uncertainty is provided by the renormalization scheme ambiguity for the bottom-quark mass, which we estimated by varying from the on-shell to the $\overline{\mathrm{MS}}$ scheme. This amounts to an uncertainty of up to $20 \%$ and it dominates the error budget of our prediction for the top-bottom interference at small values of the Higgs $p_{\perp}$. Together with the uncertainties associated with the fixed order calculation, we also performed a detailed study of the ones associated with the resummation procedure in the presence of bottom quarks. In order to estimate these ambiguities for the top-bottom interference, we matched the fixed order NLO predictions with the NNLL resummed crosssection using two different schemes, an additive and a multiplicative one, and two very different choices of the resummation scale, $Q_{b}=2 m_{b}$ and $Q_{b}=m_{H} / 2$. This leads to an uncertainty between $15-20 \%$ on the top-bottom interference contribution to the $p_{\perp}$ spectrum. Since the interference amounts to about $5 \%$ of the full $p_{\perp}$ spectrum, we conclude that unknown higher order $b$-quark mass effects can modify the Higgs transverse momentum distribution by few percent. All ambiguities associated with the resummation in the presence of bottom quarks produce consistent results within the NNLL+NLO uncertainty band, which is however driven by uncertainties in the (NLO) top quark contribution. The latter is currently known to higher $\mathrm{N}^{3} \mathrm{LL}+\mathrm{NNLO}$ accuracy $[7-11,29,55]$. It would be interesting to combine these results with the ones presented in this article. We leave this for future investigations.

In conclusion, we presented a description of the Higgs $p_{\perp}$ spectrum at NNLL+NLO QCD including both top and bottom quark contributions. We found that the uncertainty on the top-bottom interference is $\mathcal{O}(20 \%)$ in the region of interest $m_{b} \lesssim p_{\perp} \lesssim m_{H}$. Given the intrinsic ambiguities from scale dependence and, in particular, from the choice of the bottom-mass renormalization scheme and matching scheme, any improvement in this description will inevitably require the computation of the NNLO QCD corrections to the bottom-quark contribution to $g g \rightarrow H$ and $g g \rightarrow H+$ jet.

\section{Acknowledgments}

Some of us would like to thank the Munich Institute for Astronomy and Particle Physics (MIAPP) for hospitality and partial support during the programs Automated, Resummed and Effective (F.C., K.M, P.F.M., L.T. and C.W.) and Mathematics and Physics of Scattering Amplitudes (F.C., L.T. and C.W.). The work of F.C. and L.T. was supported in part by the ERC starting grant 637019 "MathAm". The research of K.M. is partially supported by BMBF grant 05H15VKCCA. P.F.M. has been supported by a Marie Skłodowska 
Curie Individual Fellowship of the European Commission's Horizon 2020 Programme under contract number 702610 Resummation4PS.

\section{A Resummation and matching: details}

In this appendix we briefly derive the resummation formula in the toy model described in the main text, report the final NNLL formulas that we eventually used in our results and describe in details the matching procedure we employ. We follow the approach of refs. $[29,55]$, and we refer the reader to these publications for the details.

\section{A.1 The LL case}

We consider the $p_{\perp}$ distribution of a Higgs boson in $p p \rightarrow H$ in the $p_{\perp} \rightarrow 0$ limit, at leading-logarithmic accuracy. In this approximation, one must only control the leading singularity of the $n$-emissions matrix element at all perturbative orders. This is done by approximating the process with an ensemble of independent soft-collinear gluons emitted off the two incoming legs.

To set up the notation we introduce two reference light-like momenta along the beam direction that will serve to parametrize the radiation ${ }^{16}$

$$
\tilde{p}_{1}=\frac{m_{H}}{2}(1,0,0,1), \quad \tilde{p}_{2}=\frac{m_{H}}{2}(1,0,0,-1) .
$$

We now consider a real emission $k_{1}$ collinear to $\tilde{p}_{1}$ that can be expressed as

$$
k_{1}=\left(1-z_{1}^{\left(\ell_{1}\right)}\right) \tilde{p}_{1}+\left(1-z_{1}^{\left(\ell_{2}\right)}\right) \tilde{p}_{2}+\kappa_{\perp 1}
$$

where $\kappa_{\perp 1}$ is a space-like four-vector, orthogonal to both $\tilde{p}_{1}$ and $\tilde{p}_{2}$ such that $\kappa_{\perp 1}^{2}=-k_{\perp 1}^{2}$. Note that since $k_{1}$ is massless

$$
k_{\perp 1}^{2}=\left(1-z_{1}^{\left(\ell_{1}\right)}\right)\left(1-z_{1}^{\left(\ell_{2}\right)}\right) m_{H}^{2}=\frac{2\left(\tilde{p}_{1} \cdot k_{1}\right) 2\left(\tilde{p}_{2} \cdot k_{1}\right)}{2\left(\tilde{p}_{1} \cdot \tilde{p}_{2}\right)} .
$$

Moreover, if $k_{1}$ is collinear to $\tilde{p}_{1}$ one has (from $\left(1-z_{1}^{\left(\ell_{1}\right)}\right)>\left(1-z_{1}^{\left(\ell_{2}\right)}\right)$ )

$$
z_{1}^{\left(\ell_{1}\right)}<1-\frac{k_{\perp 1}}{m_{H}}
$$

An analogous limit on $z_{1}^{\left(\ell_{2}\right)}$ as in eq. (A.3) holds when $k_{1}$ is collinear to $\tilde{p}_{2}$. Subsequent emissions off leg $\ell_{1}$ can be parametrized analogously to eq. (A.2), replacing the reference momentum $\tilde{p}_{1}$ with

$$
\tilde{p}_{1} \rightarrow\left(\prod_{\substack{i=1 \\ \ell_{i}=\ell_{1}}}^{k} z_{i}^{\left(\ell_{i}\right)}\right) \tilde{p}_{1} \simeq \tilde{p}_{1}
$$

\footnotetext{
${ }^{16}$ We remind the reader that we are working in the soft approximation. As a consequence the kinematics is much simplified.
} 
where the product runs over all emissions off leg $\ell_{1}$ that occur prior to the emission we are parametrizing, and we used the fact that in the soft limit $z_{i}^{\left(\ell_{i}\right)} \simeq 1$. A similar parametrization holds for emissions off leg $\ell_{2}$.

The transverse recoil of the radiation is absorbed entirely by the Higgs boson that acquires a transverse momentum

$$
p_{\perp}=\left|\sum_{i} \vec{k}_{\perp i}\right|
$$

In order to predict the $p_{\perp} \rightarrow 0$ limit, we need to sum emissions at all orders in the strong coupling. With LL accuracy, the squared amplitude for $n$ emissions can be approximated by a product of $n$ independent splitting kernels, as the soft correlation between emissions starts contributing at NLL order. The physical picture corresponding to this approximation is given by a set of independent emissions off legs $\ell_{1}$ and $\ell_{2}$. In this approximation, the differential partonic distribution can be written as

$$
\begin{aligned}
\frac{\mathrm{d} \hat{\sigma}}{\mathrm{d} p_{\perp}} \simeq & {\left[d p_{H}\right]\left|\mathcal{M}\left(\tilde{p}_{1}+\tilde{p}_{2} \rightarrow H\right)\right|^{2} \delta^{(4)}\left(\tilde{p}_{1}+\tilde{p}_{2}-p_{H}\right) } \\
& \times \frac{1}{n !} \prod_{i=1}^{n}\left[d k_{i}\right]\left|M_{\text {soft }}\left(k_{i}\right)\right|^{2} \delta\left(p_{\perp}-\left|\sum_{i} \vec{k}_{\perp i}\right|\right),
\end{aligned}
$$

where the eikonal squared amplitude for a single emission reads

$$
[d k]\left|M_{\mathrm{soft}}(k)\right|^{2}=\sum_{\ell=1,2} 2 C_{A} \frac{\alpha_{s}\left(k_{\perp}\right)}{\pi} \frac{d k_{\perp}}{k_{\perp}} \frac{d z^{(\ell)}}{1-z^{(\ell)}} \Theta\left(\left(1-z^{(\ell)}\right)-k_{\perp} / m_{H}\right) \frac{d \phi}{2 \pi} .
$$

In eq. (A.7), the coupling is evaluated at $k_{\perp}$ to account for the leading-logarithmic contribution of the gluon branching into either a pair of soft quarks or gluons, see e.g. [56] for a detailed explanation.

The resummation is naturally performed at the level of the cumulative distribution, defined as

$$
\Sigma\left(p_{\perp}\right)=\int_{0}^{p_{\perp}} d p_{\perp}^{\prime} \frac{d \sigma}{d p_{\perp}^{\prime}} .
$$

Indeed while the differential spectrum involves plus distributions in $p_{\perp}, \Sigma\left(p_{\perp}\right)$ is a regular function. From eq. (A.6), it follows that the cumulative distribution with LL accuracy can be written as

$$
\Sigma\left(p_{\perp}\right) \simeq\left[f_{g}\left(\mu_{F}\right) \otimes f_{g}\left(\mu_{F}\right)\right]\left(m_{H}^{2} / s\right) \times \int d \hat{\sigma} \Theta\left(p_{\perp}-\left|\sum_{i} \vec{k}_{\perp i}\right|\right),
$$

where $f_{g}\left(\mu_{F}\right)$ is the gluon parton density evaluated at the factorization scale $\mu_{F}$, and the convolution is defined as usual

$$
[f \otimes g](x) \equiv \int_{0}^{1} \mathrm{~d} y \mathrm{~d} z \delta(x-y z) f(y) g(z) .
$$


Since $p_{\perp}$ only constrains the transverse momentum of the emissions, we can perform the integrals over the $z_{i}^{\left(\ell_{i}\right)}$ components inclusively. It is therefore convenient to introduce the functions

$$
\begin{aligned}
& R_{1}^{\prime}\left(p_{\perp}\right)=\int[d k]\left|M_{\mathrm{soft}}(k)\right|^{2}(2 \pi) \delta(\phi-\bar{\phi}) p_{\perp} \delta\left(p_{\perp}-k_{\perp}\right) \Theta\left(z^{(2)}-z^{(1)}\right) \\
& R_{2}^{\prime}\left(p_{\perp}\right)=\int[d k]\left|M_{\mathrm{soft}}(k)\right|^{2}(2 \pi) \delta(\phi-\bar{\phi}) p_{\perp} \delta\left(p_{\perp}-k_{\perp}\right) \Theta\left(z^{(1)}-z^{(2)}\right) .
\end{aligned}
$$

This notation allows us to parametrize the real-emission matrix element and phase space as

$$
\left[d k_{i}\right]\left|M_{\text {soft }}\left(k_{i}\right)\right|^{2}=\frac{d k_{\perp i}}{k_{\perp i}} \frac{d \phi_{i}}{2 \pi} \sum_{\ell_{i}=1,2} R_{\ell_{i}}^{\prime}\left(k_{\perp i}\right)=\frac{d \zeta_{i}}{\zeta_{i}} \frac{d \phi_{i}}{2 \pi} \sum_{\ell_{i}=1,2} R_{\ell_{i}}^{\prime}\left(\zeta_{i} k_{\perp 1}\right),
$$

where we defined $\zeta_{i}=k_{\perp i} / k_{\perp 1}$.

We now discuss the purely virtual corrections, which are encoded in the gluon form factor $\left|\mathcal{M}\left(\tilde{p}_{1}+\tilde{p}_{2} \rightarrow H\right)\right|^{2}$. We write it as

$$
\left|\mathcal{M}\left(\tilde{p}_{1}+\tilde{p}_{2} \rightarrow H\right)\right|^{2}=\mathcal{H}\left(m_{H}\right)\left|\mathcal{M}_{B}\left(\tilde{p}_{1}+\tilde{p}_{2} \rightarrow H\right)\right|^{2},
$$

where the function $\mathcal{H}$ contains all the IRC singularities and the constant finite corrections of the form factor, and $\mathcal{M}_{B}$ denotes the Born amplitude. Since we are working with LL accuracy, we are only interested in the leading singular term of $\mathcal{H}$ at all orders (while neglecting all finite terms) which can be written as

$$
\mathcal{H}\left(m_{H}\right) \simeq \exp \left\{-\int[d k]\left|M_{\text {soft }}(k)\right|^{2}\right\} .
$$

Note that the integral in eq. (A.14) is divergent and is to be considered as regularized. In order to cancel the IRC divergences of the real emissions (A.6) against the ones in the virtual corrections (A.14) at all orders, we introduce a small slicing parameter $\epsilon>0$ such that all emissions with a transverse momentum $k_{\perp i}$ smaller than $\epsilon k_{\perp 1}$ can be ignored in the computation of the observable $p_{\perp}$, in the limit $\epsilon \rightarrow 0$. The real emissions with $k_{\perp i}<\epsilon k_{\perp 1}$, hereby denoted as unresolved, can be directly combined with the virtual corrections at all orders. Their combination gives rise to an exponential suppression factor of the type

$$
\begin{aligned}
\mathcal{H}\left(m_{H}\right) & \sum_{m}^{\infty} \frac{1}{m !} \prod_{i=1}^{m}\left[\int \frac{d k_{\perp i}}{k_{\perp i}} \frac{d \phi_{i}}{2 \pi} \sum_{\ell_{i}=1,2} R_{\ell_{i}}^{\prime}\left(k_{\perp i}\right) \Theta\left(\epsilon k_{\perp 1}-k_{\perp i}\right)\right] \\
= & \exp \left\{-\int \frac{d k_{\perp}}{k_{\perp}} \frac{d \phi}{2 \pi} \sum_{\ell=1,2} R_{\ell}^{\prime}\left(k_{\perp}\right) \Theta\left(k_{\perp}-\epsilon k_{\perp 1}\right)\right\} \equiv e^{-R\left(\epsilon k_{\perp 1}\right)} .
\end{aligned}
$$

On the other end, emissions with $k_{\perp i}>\epsilon k_{\perp 1}$, that we denote as resolved, are constrained by the observable's measurement function and therefore cannot be integrated over inclusively. The resummed LL cross section thus reads

$$
\begin{aligned}
\Sigma\left(p_{\perp}\right) \simeq & \sigma_{B} \int \frac{d k_{\perp 1}}{k_{\perp 1}} \frac{d \phi_{1}}{2 \pi} e^{-R\left(\epsilon k_{\perp 1}\right)} \sum_{\ell_{1}=1,2} R_{\ell_{1}}^{\prime}\left(k_{\perp 1}\right) \\
& \times \sum_{n=0}^{\infty} \frac{1}{n !} \prod_{i=2}^{n+1} \int_{\epsilon}^{1} \frac{d \zeta_{i}}{\zeta_{i}} \frac{d \phi_{i}}{2 \pi} \sum_{\ell_{i}=1,2} R_{\ell_{i}}^{\prime}\left(\zeta_{i} k_{\perp 1}\right) \Theta\left(p_{\perp}-\left|\sum_{i} \vec{k}_{\perp i}\right|\right),
\end{aligned}
$$


where $\sigma_{B}$ is the Born cross section. The above formula, in the limit $\epsilon \rightarrow 0$ exactly reproduces the LL corrections to the $p_{\perp}$ distribution, see ref. [29] for a formal proof. Eq (A.16) can be further simplified by observing that in the resolved radiation one always has $\zeta_{i} \sim 1$, since configurations in which $\zeta_{i} \ll 1$ are automatically canceled against the exponential Sudakov factor $e^{-R\left(\epsilon k_{\perp 1}\right)}$. Therefore, one can expand the functions $R^{\prime}\left(\zeta_{i} k_{\perp 1}\right)$ in powers of $\ln \left(1 / \zeta_{i}\right)$ as

$$
R_{\ell_{i}}^{\prime}\left(\zeta_{i} k_{\perp 1}\right)=R_{\ell_{i}}^{\prime}\left(k_{\perp 1}\right)+R_{\ell_{i}}^{\prime \prime}\left(k_{\perp 1}\right) \ln \frac{1}{\zeta_{i}}+\ldots,
$$

and retain terms that contribute at a given logarithmic order. In particular, at LL, only the first term in this expansion contributes, and higher-order terms matter at higher logarithmic orders (see refs. [29] for details).

Similarly, we can consistently expand out the $\epsilon$ dependence of the exponential Sudakov as

$$
e^{-R\left(\epsilon k_{\perp 1}\right)}=e^{-R\left(k_{\perp 1}\right)} e^{-R^{\prime}\left(k_{\perp 1}\right) \ln \frac{1}{\epsilon}+\ldots},
$$

where the $\epsilon$ dependence manifestly cancels against the one in the resolved contribution, and we defined

$$
R^{\prime}\left(k_{\perp 1}\right) \equiv \sum_{\ell_{1}=1,2} R_{\ell_{1}}^{\prime}\left(k_{\perp 1}\right) .
$$

Therefore, with LL accuracy, eq. (A.16) becomes

$$
\begin{aligned}
\Sigma\left(p_{\perp}\right) \simeq & \sigma_{B} \int \frac{d k_{\perp 1}}{k_{\perp 1}} \frac{d \phi_{1}}{2 \pi} e^{-R\left(k_{\perp 1}\right)} \epsilon^{R^{\prime}\left(k_{\perp 1}\right)} \sum_{\ell_{1}=1,2} R_{\ell_{1}}^{\prime}\left(k_{\perp 1}\right) \\
& \times \sum_{n=0}^{\infty} \frac{1}{n !} \prod_{i=2}^{n+1} \int_{\epsilon}^{1} \frac{d \zeta_{i}}{\zeta_{i}} \frac{d \phi_{i}}{2 \pi} \sum_{\ell_{i}=1,2} R_{\ell_{i}}^{\prime}\left(k_{\perp 1}\right) \Theta\left(p_{\perp}-\left|\sum_{i} \vec{k}_{\perp i}\right|\right) .
\end{aligned}
$$

Equation (A.20) is suitable for a numerical implementation, as explained in ref. [29] in detail. The dependence on $\epsilon$ is at most power suppressed (i.e. $\mathcal{O}\left(\epsilon p_{\perp}\right)$ ) and it vanishes in the limit $\epsilon \rightarrow 0$. This limit can therefore be taken safely numerically, and the result is absolutely stable for very small values of $\epsilon{ }^{17}$

We now introduce the resummation scale $Q$ as a possible way to switch off the resummation at large transverse momentum. This is defined with a procedure similar to the one discussed in the text. We first break the logarithm as follows

$$
L \equiv \ln \frac{m_{H}}{k_{\perp 1}}=\ln \frac{m_{H}}{Q}+\ln \frac{Q}{k_{\perp 1}} .
$$

The above operation will allow us (as explained shortly) to have an additional handle (namely the scale $Q$ ) to estimate the size of subleading logarithmic terms. Moreover, we also slightly modify the phase space available for the radiation, by introducing powersuppressed contributions that ensure that at large $p_{\perp}$ the resummation effects completely

\footnotetext{
${ }^{17}$ In our implementation we use $\epsilon=e^{-20}$, although any value below $\epsilon=e^{-6}$ does not lead to any appreciable differences.
} 
vanish. This can be done, as a first step, by modifying the resummed logarithms as follows

$$
\ln \frac{Q}{k_{\perp 1}} \rightarrow \frac{1}{p} \ln \left(\frac{Q^{p}}{k_{\perp 1}^{p}}+1\right) \equiv \tilde{L}
$$

where $p$ is a positive real parameter which is chosen such that the resummed differential distribution vanishes as $1 / p_{\perp}^{p+1}$ at large $p_{\perp}$. The above prescription essentially amounts to the following

1. First, we split the resummed $\operatorname{logarithm} L$ into the sum of a small $\operatorname{logarithm} \ln \left(m_{H} / Q\right)$ (with $Q \sim m_{H}$ ) and a large one $\ln \left(Q / k_{\perp 1}\right)$. This operation allows one to introduce a generic scale $Q$ which appears in the resummed logarithms. One can now expand $L$ about $\ln \left(Q / k_{\perp 1}\right)$, retaining all terms with the desired logarithmic accuracy. Effectively, this implies that $\ln \left(m_{H} / Q\right)$ is treated perturbatively at fixed order. Moreover, we replace $\ln \left(Q / k_{\perp 1}\right)$ by the modified logarithm $\tilde{L}$. In our LL example this means

$$
R\left(k_{\perp 1}\right) \rightarrow \tilde{R}\left(k_{\perp 1}\right)+\mathcal{O}\left(\ln m_{H} / Q\right) ; \quad R\left(k_{\perp 1}\right) \rightarrow \tilde{R}^{\prime}\left(k_{\perp 1}\right)+\mathcal{O}\left(\ln m_{H} / Q\right),
$$

where $\tilde{R}$ and $\tilde{R}^{\prime}$ are functions of the modified logarithm $\tilde{L}$ only.

2. eq. (A.22) comes together with the following prefactor $\mathcal{J}$ in eq. (A.20)

$$
\mathcal{J}\left(k_{\perp 1}\right)=\left(\frac{Q}{k_{\perp 1}}\right)^{p}\left(1+\left(\frac{Q}{k_{\perp 1}}\right)^{p}\right)^{-1} .
$$

This corresponds to the Jacobian for the transformation (A.22), and ensures the absence of fractional (although power suppressed) $\alpha_{s}$ powers in the final distribution [29]. This factor, once again, leaves the small $k_{\perp 1}$ region untouched, and only modifies the large $p_{\perp}$ region by power-suppressed effects. This is effectively mapping the limit $k_{\perp 1} \rightarrow Q$ onto $k_{\perp 1} \rightarrow \infty$. Although this procedure seems a simple change of variables, we stress that the observable's measurement function (i.e. the $\Theta$ function in eq. (A.20)) is not affected by this prescription. As a consequence, the final result will depend on the parameter $p$ through power-suppressed terms.

The difference between the above prescription and what was introduced in the text is that the argument of the (modified) logarithms is now $k_{\perp 1}$ instead of $p_{\perp}$. This prescription is technically more correct, since in the small $k_{\perp 1}$ region, which governs the $p_{\perp} \rightarrow 0$ limit, the modified logarithms leave eq. (A.20) untouched. Conversely, at large $k_{\perp 1}$, where one has $k_{\perp 1} \sim p_{\perp}$, the above prescription reduces to what was defined in the text, i.e. the modified logarithms of $k_{\perp 1}$ in this region are formally equivalent to modified logarithms in $p_{\perp}$. To see this, we observe that when $k_{\perp 1} \gg Q$ the function $R^{\prime}\left(k_{\perp 1}\right) \ll 1$. Therefore, the probability of having any emission after the first one in eq. (A.20) is strongly suppressed. As a consequence, at large $k_{\perp 1}$, the only relevant event is the one that involves a single emission $k_{1}$, for which the cross section reads

$$
\Sigma\left(p_{\perp}\right) \sim \sigma_{B} \int \frac{d k_{\perp 1}}{k_{\perp 1}} \frac{d \phi_{1}}{2 \pi} \mathcal{J}\left(k_{\perp 1}\right) e^{-\tilde{R}\left(k_{\perp 1}\right)} \sum_{\ell_{1}=1,2} \tilde{R}_{\ell_{1}}^{\prime}\left(k_{\perp 1}\right) \Theta\left(p_{\perp}-\left|\vec{k}_{\perp 1}\right|\right)=e^{-\tilde{R}\left(p_{\perp}\right)} .
$$


It is easy to see that, if eq. (A.25) were evaluated without the factor $\mathcal{J}$, it would lead to additional power-suppressed terms with fractional power of the coupling, which are clearly spurious.

\section{A.2 Final formulas for NNLL resummation}

Beyond LL, eq. (A.20) is corrected to account for the description of the real-emission matrix element and phase space in less singular configurations, as well as higher perturbative corrections. To NNLL order it can be expressed as [29]

$$
\begin{aligned}
\Sigma\left(p_{\perp}\right)= & \int \frac{d k_{\perp 1}}{k_{\perp 1}} \frac{d \phi_{1}}{2 \pi} \mathcal{J}\left(k_{\perp 1}\right)\left[\epsilon^{\tilde{R}^{\prime}\left(k_{\perp 1}\right)} \sum_{n=0}^{\infty} \frac{1}{n !} \prod_{i=2}^{n+1} \int_{\epsilon}^{1} \frac{d \zeta_{i}}{\zeta_{i}} \frac{d \phi_{i}}{2 \pi} \sum_{\ell_{1}=1,2} \tilde{R}_{\ell_{1}}^{\prime}\left(k_{\perp 1}\right)\right] \\
& \times\left\{\frac{d}{d \tilde{L}}\left[-e^{-\tilde{R}\left(k_{\perp 1}\right)} \mathcal{L}\left(k_{\perp 1}\right)\right] \Theta\left(p_{\perp}-\left|\sum_{i=1}^{n+1} \vec{k}_{\perp i}\right|\right)+e^{-\tilde{R}\left(k_{\perp 1}\right)} \tilde{R}^{\prime}\left(k_{\perp 1}\right)\right. \\
& \times \int_{\epsilon}^{1} \frac{d \zeta_{s}}{\zeta_{s}} \frac{d \phi_{s}}{2 \pi}\left[\sum_{\ell_{i}=1,2}\left(\delta \tilde{R}_{\ell_{i}}^{\prime}\left(k_{\perp 1}\right)+\tilde{R}_{\ell_{i}}^{\prime \prime}\left(k_{\perp 1}\right) \ln \frac{k_{\perp 1}}{k_{\perp s}}\right) \hat{\mathcal{L}}\left(k_{\perp 1}\right)-\frac{d \hat{\mathcal{L}}\left(k_{\perp 1}\right)}{d \tilde{L}}\right] \\
& \left.\times\left[\Theta\left(p_{\perp}-\left|\sum_{i=1}^{n+2} \vec{k}_{\perp i}\right|\right)-\Theta\left(p_{\perp}-\left|\sum_{\substack{i=1 \\
i \neq s}}^{n+2} \vec{k}_{\perp i}\right|\right)\right]\right\},
\end{aligned}
$$

where $\zeta_{i}=k_{\perp i} / k_{\perp 1}$. In this formula, the Sudakov radiator $\tilde{R}\left(k_{\perp 1}\right)$ is corrected with respect to its LL expression by higher-order corrections of both soft and collinear origin. The same comment applies to the function $\tilde{R}_{\ell_{i}}^{\prime}$ which we decided to split into the old $\tilde{R}^{\prime}$ (derivative of the LL radiator defined above), plus a correction that contains all subleading effects, therefore replacing $\tilde{R}^{\prime}$ with

$$
\tilde{R}_{\ell_{i}}^{\prime} \rightarrow \tilde{R}_{\ell_{i}}^{\prime}+\delta \tilde{R}_{\ell_{i}}^{\prime}
$$

The correction due to $\delta \tilde{R}_{\ell_{i}}^{\prime}$ is only relevant to NNLL order for one of the resolved emissions. This special emission is denoted by the subscript $s$ in eq. (A.26). After expanding to first order the corresponding term proportional to $\delta \tilde{R}_{\ell_{i}}^{\prime}$ arising from the initial $\epsilon^{\tilde{R}^{\prime}}$ factor, one ends up with the second term in the curly bracket in eq. (A.26), see ref. [29] for a full derivation. The same manipulations apply to the $\tilde{R}^{\prime \prime}$ correction coming from the expansion (A.17) discussed above.

Moreover, we introduced the following generalized luminosity coefficient

$$
\begin{aligned}
\mathcal{L}\left(k_{\perp 1}\right)= & \left|\mathcal{M}_{B}\right|^{2} \sum_{i, j} \int d x_{1} d x_{2} \int_{x_{1}}^{1} \frac{d z_{1}}{z_{1}} \int_{x_{2}}^{1} \frac{d z_{2}}{z_{2}} f_{i}\left(\mu_{F} e^{-\tilde{L}}, \frac{x_{1}}{z_{1}}\right) f_{j}\left(\mu_{F} e^{-\tilde{L}}, \frac{x_{2}}{z_{2}}\right) \\
& \times \delta\left(x_{1} x_{2} s-m_{H}^{2}\right)\left[\delta_{g i} \delta_{g j} \delta\left(1-z_{1}\right) \delta\left(1-z_{2}\right)\left(1+\frac{\alpha_{s}\left(\mu_{R}\right)}{2 \pi} \tilde{H}^{(1)}\left(\mu_{R}, \frac{Q}{m_{H}}\right)\right)\right. \\
& \left.+\frac{\alpha_{s}\left(\mu_{R}\right)}{2 \pi} \frac{1}{1-2 \alpha_{s}\left(\mu_{R}\right) \beta_{0} \tilde{L}}\left(\tilde{C}_{g i}^{(1)}\left(z_{1}, \mu_{F}, \frac{Q}{m_{H}}\right) \delta\left(1-z_{2}\right) \delta_{g j}+\left\{z_{1} \leftrightarrow z_{2} ; i \leftrightarrow j\right\}\right)\right],
\end{aligned}
$$


and its NLL approximation

$$
\hat{\mathcal{L}}\left(k_{\perp 1}\right)=\left|\mathcal{M}_{B}\right|^{2} \int d x_{1} d x_{2} f_{g}\left(\mu_{F} e^{-\tilde{L}}, x_{1}\right) f_{g}\left(\mu_{F} e^{-\tilde{L}}, x_{2}\right) \delta\left(x_{1} x_{2} s-m_{H}^{2}\right) .
$$

We now report all the various ingredients entering the above formulas. The $\mathcal{O}\left(\alpha_{s}\right)$ correction to the collinear coefficient functions reads

$$
\tilde{C}_{g i}^{(1)}\left(z, \mu_{F}, \frac{Q}{m_{H}}\right)=-P_{i j}^{(0), \epsilon}(z)-\delta_{i j} \delta(1-z) C_{A} \frac{\pi^{2}}{12}+P_{i j}^{(0)}(z) \ln \frac{Q^{2}}{\mu_{F}^{2}}
$$

where $P_{i j}^{(0)}$ are the LO Altarelli-Parisi splitting functions

$$
\begin{aligned}
& P_{q q}^{(0)}(z)=C_{F}\left[\frac{1+z^{2}}{(1-z)_{+}}+\frac{3}{2} \delta(1-z)\right], \\
& P_{q g}^{(0)}(z)=T_{R}\left[z^{2}+(1-z)^{2}\right], \\
& P_{g q}^{(0)}(z)=C_{F} \frac{1+(1-z)^{2}}{z}, \\
& P_{g g}^{(0)}(z)=2 C_{A}\left[\frac{z}{(1-z)_{+}}+\frac{1-z}{z}+z(1-z)\right]+2 \pi \beta_{0} \delta(1-z),
\end{aligned}
$$

with $\beta_{0}=\left(11 C_{A}-2 n_{f}\right) /(12 \pi)$ and $P_{i j}^{(0), \epsilon}(z)$ are given by

$$
\begin{aligned}
& P_{q q}^{(0), \epsilon}(z)=-C_{F}(1-z), \\
& P_{g q}^{(0), \epsilon}(z)=-C_{F} z, \\
& P_{q g}^{(0), \epsilon}(z)=-2 T_{R} z(1-z), \\
& P_{g g}^{(0), \epsilon}(z)=0 .
\end{aligned}
$$

The function $\tilde{H}^{(1)}\left(\mu_{R}, \frac{Q}{m_{H}}\right)$ is defined as

$$
\tilde{H}^{(1)}\left(\mu_{R}, \frac{Q}{m_{H}}\right)=H^{(1)}-\left(B^{(1)}+\frac{A^{(1)}}{2} \ln \frac{m_{H}^{2}}{Q^{2}}\right) \ln \frac{m_{H}^{2}}{Q^{2}}+4 \pi \beta_{0} \ln \frac{\mu_{R}^{2}}{m_{H}{ }^{2}},
$$

where $H^{(1)}$ denotes the finite one-loop virtual correction to the $g g \rightarrow H$ process and $A^{(i)}, B^{(i)}$ are reported below. For the top contribution in the $m_{t} \rightarrow \infty$ approximation, $H^{(1)}$ reads

$$
H^{(1)}=C_{A}\left(5+\frac{7}{6} \pi^{2}\right)-3 C_{F}=11+\frac{7}{2} \pi^{2}
$$

The result including full quark mass dependence has been computed analytically in refs. [45, $57,58] .{ }^{18}$

We expand the Sudakov radiator as

$$
\tilde{R}=\tilde{L} g_{1}(\lambda)+g_{2}(\lambda)+\frac{\alpha_{s}\left(m_{H}\right)}{\pi} g_{3}(\lambda)
$$

\footnotetext{
${ }^{18}$ In our implementation we take both the Born amplitude and the virtual corrections from ref. [45].
} 
where

$$
\lambda=\alpha_{s}\left(\mu_{R}\right) \beta_{0} \tilde{L}
$$

We introduce

$$
x_{Q}=\frac{Q}{m_{H}},
$$

and write

$$
\begin{aligned}
g_{1}(\lambda)= & \frac{A^{(1)}}{\pi \beta_{0}} \frac{2 \lambda+\ln (1-2 \lambda)}{2 \lambda}, \\
g_{2}(\lambda)= & \frac{1}{2 \pi \beta_{0}} \ln (1-2 \lambda)\left(A^{(1)} \ln \frac{1}{x_{Q}^{2}}+B^{(1)}\right)-\frac{A^{(2)}}{4 \pi^{2} \beta_{0}^{2}} \frac{2 \lambda+(1-2 \lambda) \ln (1-2 \lambda)}{1-2 \lambda} \\
& +A^{(1)}\left(-\frac{\beta_{1}}{4 \pi \beta_{0}^{3}} \frac{\ln (1-2 \lambda)((2 \lambda-1) \ln (1-2 \lambda)-2)-4 \lambda}{1-2 \lambda}\right. \\
& \left.-\frac{1}{2 \pi \beta_{0}} \frac{(2 \lambda(1-\ln (1-2 \lambda))+\ln (1-2 \lambda))}{1-2 \lambda} \ln \frac{\mu_{R}^{2}}{x_{Q}^{2} m_{H^{2}}}\right), \\
g_{3}(\lambda)= & \left(A^{(1)} \ln \frac{1}{x_{Q}^{2}}+B^{(1)}\right)\left(-\frac{\lambda}{1-2 \lambda} \ln \frac{\mu_{R}^{2}}{x_{Q}^{2} m_{H}^{2}}+\frac{\beta_{1}}{2 \beta_{0}^{2}} \frac{2 \lambda+\ln (1-2 \lambda)}{1-2 \lambda}\right) \\
& -\frac{1}{2 \pi \beta_{0}} \frac{\lambda}{1-2 \lambda}\left(A^{(2)} \ln \frac{1}{x_{Q}^{2}}+B^{(2)}\right)-\frac{A^{(3)}}{4 \pi^{2} \beta_{0}^{2}} \frac{\lambda^{2}}{(1-2 \lambda)^{2}} \\
& +A^{(2)}\left(\frac{\beta_{1}}{4 \pi \beta_{0}^{3}} \frac{2 \lambda(3 \lambda-1)+(4 \lambda-1) \ln (1-2 \lambda)}{(1-2 \lambda)^{2}}-\frac{1}{\pi \beta_{0}} \frac{\lambda^{2}}{(1-2 \lambda)^{2}} \ln \frac{\mu_{R}^{2}}{x_{Q}^{2} m_{H}^{2}}\right) \\
& +A^{(1)}\left(\frac{\lambda\left(\beta_{0} \beta_{2}(1-3 \lambda)+\beta_{1}^{2} \lambda\right)}{\beta_{0}^{4}(1-2 \lambda)^{2}}+\frac{(1-2 \lambda) \ln (1-2 \lambda)\left(\beta_{0} \beta_{2}(1-2 \lambda)+2 \beta_{1}^{2} \lambda\right)}{2 \beta_{0}^{4}(1-2 \lambda)^{2}}\right. \\
& +\frac{\beta_{1}^{2}}{4 \beta_{0}^{4}} \frac{(1-4 \lambda) \ln { }^{2}(1-2 \lambda)}{(1-2 \lambda)^{2}}-\frac{\lambda^{2}}{(1-2 \lambda)^{2}} \ln { }^{2} \frac{\mu_{R}^{2}}{x_{Q}^{2} m_{H^{2}}} \\
& \left.-\frac{\beta_{1}}{2 \beta_{0}^{2}} \frac{(2 \lambda(1-2 \lambda)+(1-4 \lambda) \ln (1-2 \lambda))}{(1-2 \lambda)^{2}} \frac{\mu_{R}^{2}}{x_{Q}^{2} m_{H}^{2}}\right) .
\end{aligned}
$$

The expressions of $\tilde{R}^{\prime}, \delta \tilde{R}^{\prime}$, and $\tilde{R}^{\prime \prime}$ used in eq. (A.26) are defined as

$$
\tilde{R}^{\prime}=-\frac{d}{d \tilde{L}}\left(\tilde{L} g_{1}(\lambda)\right), \quad \delta \tilde{R}^{\prime}=-\frac{d g_{2}(\lambda)}{d \tilde{L}}, \quad \tilde{R}^{\prime \prime}=\frac{d \tilde{R}^{\prime}}{d \tilde{L}}
$$

The $\beta$ function coefficients read

$$
\begin{aligned}
& \beta_{0}=\frac{11 C_{A}-2 n_{f}}{12 \pi}, \\
& \beta_{1}=\frac{17 C_{A}^{2}-5 C_{A} n_{f}-3 C_{F} n_{f}}{24 \pi^{2}}, \\
& \beta_{2}=\frac{2857 C_{A}^{3}+\left(54 C_{F}^{2}-615 C_{F} C_{A}-1415 C_{A}^{2}\right) n_{f}+\left(66 C_{F}+79 C_{A}\right) n_{f}^{2}}{3456 \pi^{3}} .
\end{aligned}
$$


Finally, we have

$$
\begin{aligned}
A^{(1)}= & 2 C_{A}, \\
A^{(2)}= & \left(\frac{67}{9}-\frac{\pi^{2}}{3}\right) C_{A}^{2}-\frac{10}{9} C_{A} n_{f}, \\
A^{(3)}= & \left(-22 \zeta_{3}-\frac{67 \pi^{2}}{27}+\frac{11 \pi^{4}}{90}+\frac{15503}{324}\right) C_{A}^{3}+\left(\frac{10 \pi^{2}}{27}-\frac{2051}{162}\right) C_{A}^{2} n_{f} \\
& +\left(4 \zeta_{3}-\frac{55}{12}\right) C_{A} C_{F} n_{f}+\frac{50}{81} C_{A} n_{f}^{2}, \\
B^{(1)}= & -\frac{11}{3} C_{A}+\frac{2}{3} n_{f}, \\
B^{(2)}= & \left(\frac{11 \zeta_{2}}{6}-6 \zeta_{3}-\frac{16}{3}\right) C_{A}^{2}+\left(\frac{4}{3}-\frac{\zeta_{2}}{3}\right) C_{A} n_{f}+C_{A} C_{F} .
\end{aligned}
$$

\section{A.3 Matching to fixed order}

In this section we discuss the matching of the resummed and the fixed-order results. We work at the level of the cumulative distribution $\Sigma$, that at NNLO reads

$$
\Sigma^{\mathrm{NNLO}}\left(p_{\perp}\right)=\sigma_{\mathrm{tot}}^{\mathrm{NNLO}}-\int_{p_{\perp}}^{\infty} \mathrm{d} p_{\perp}^{\prime}\left[\frac{\mathrm{d} \sigma}{\mathrm{d} p_{\perp}}\right]^{\mathrm{NLO}} .
$$

We stress that in the main text we only show results for the differential $p_{\perp}$ distribution, therefore we label them as NLO. This corresponds to what we label as NNLO at the integrated level in this appendix. Since the total $g g \rightarrow H$ cross section is not known in the full SM beyond NLO, we approximate the NNLO correction to $\sigma_{\mathrm{tot}}^{\mathrm{NNO}}$ by multiplying the exact NLO result by the NNLO/NLO $K$ factor as computed in the $m_{t} \rightarrow \infty, m_{b} \rightarrow 0$ limit. We stress, however, that at the level of the differential distributions we are interested in, this approximation is formally a $\mathrm{N}^{3} \mathrm{LL}$ effect, and it is beyond the accuracy considered in our study.

In order to assess the uncertainty associated with the matching procedure, we consider here two different matching schemes. The first scheme we introduce is the common additive scheme discussed in the main text defined as

$$
\Sigma_{\text {add }}\left(p_{\perp}\right)=\Sigma^{\mathrm{NNLL}}\left(p_{\perp}\right)+\Sigma^{\mathrm{NNLO}}\left(p_{\perp}\right)-\mathcal{T}^{\mathrm{NNLO}}\left[\Sigma^{\mathrm{NNLL}}\left(p_{\perp}\right)\right] .
$$

Since the $\mathcal{O}\left(\alpha_{s}^{2}\right)$ (relative to the Born) collinear coefficient functions and virtual corrections are unknown in the full SM, in the additive scheme we approximate them by multiplying the HEFT ones by the exact Born squared amplitude.

The second scheme we consider belongs to the class of multiplicative schemes. In the text, we schematically defined it as

$$
\Sigma_{\text {mult }}\left(p_{\perp}\right)=\Sigma^{\mathrm{NNLL}}\left(p_{\perp}\right) \mathcal{T}^{\mathrm{NNLO}}\left[\frac{\Sigma^{\mathrm{NNLO}}\left(p_{\perp}\right)}{\Sigma^{\mathrm{NNLL}}\left(p_{\perp}\right)}\right] .
$$

We recall that we indicate with $\mathcal{T}^{\mathrm{NNLO}}[f]$ the fixed-order expansion of $f$ to NNLO. The two schemes (A.46), (A.47) are equivalent at the perturbative order we are working at, as 
they only differ by $\mathrm{N}^{3} \mathrm{LO}$ and $\mathrm{N}^{3} \mathrm{LL}$ terms. The main difference between the two schemes is that, in the multiplicative approach, unlike in the additive one, higher-order corrections are damped by the resummation factor $\Sigma^{\mathrm{NNLL}}$ at low $p_{\perp}$. One advantage of the multiplicative solution is that the NNLO constant terms, of formal accuracy $\mathrm{N}^{3} \mathrm{LL}$, are automatically extracted from the fixed order in the procedure. Furthermore, as we explained in the text, in this case higher order effects introduced by the resummation follow the same scaling in $p_{\perp}$ of the fixed-order result, which at least partially mimics higher order form-factor effects.

However, there is a drawback in using eq. (A.47) as is. Indeed, $\Sigma^{\text {NNLL }}$ does not tend to one for $p_{\perp} \gg Q$, but rather to the luminosity factor defined in eq. (A.28) evaluated at $\tilde{L}=0$. Therefore, the fixed-order result $\Sigma^{\mathrm{NNLO}}$ at large $p_{\perp}$ receives a relative spurious correction of order $\alpha_{s}^{3}$

$$
\Sigma_{\text {mult }}\left(p_{\perp}\right) \sim \Sigma^{\mathrm{NNLO}}\left(p_{\perp}\right)\left(1+\mathcal{O}\left(\alpha_{s}^{3}\right)\right) .
$$

Despite being formally of higher order, these effects can be moderately sizable in processes with large $K$ factors such as Higgs production. There are different possible solutions to this problem. In ref. [29] the resummed factor (and the relative expansion) was modified by introducing a damping factor as

$$
\Sigma^{\mathrm{NNLL}} \rightarrow\left(\Sigma^{\mathrm{NNLL}}\right)^{Z}
$$

where $Z$ is a $p_{\perp}$-dependent exponent that effectively acts as a smoothened $\Theta$ function that tends to zero at large $p_{\perp}$. This solution, however, introduces new parameters that control the scaling of the damping factor $Z$ (see section 4.2 of ref. [29] for details). In this article we adopt a simpler solution, which avoids the introduction of extra parameters in the matching scheme. We therefore define the multiplicative matching scheme by normalizing the resummed prefactor to its asymptotic value for at $\tilde{L} \rightarrow 0$. This is simply given by

$$
\Sigma_{\text {asym. }}^{\mathrm{NNLL}}=\lim _{\tilde{L} \rightarrow 0} \mathcal{L}\left(k_{\perp 1}\right) .
$$

We obtain

$$
\Sigma_{\text {mult }}\left(p_{\perp}\right)=\frac{\sum^{\mathrm{NNLL}}\left(p_{\perp}\right)}{\sum_{\text {asym. }}^{\mathrm{NNLL}}} \mathcal{T}^{\mathrm{NNLO}}\left[\Sigma_{\text {asym. }}^{\mathrm{NNLL}} \cdot \frac{\sum^{\mathrm{NNLO}}\left(p_{\perp}\right)}{\Sigma^{\mathrm{NNLL}}\left(p_{\perp}\right)}\right]
$$

where

$$
\Sigma^{\mathrm{NNLL}}\left(p_{\perp}\right) \underset{p_{\perp} \gg Q}{\longrightarrow} \Sigma_{\mathrm{asym} .}^{\mathrm{NNLL}} .
$$

This ensures that in the $p_{\perp} \gg Q$ limit eq. (A.51) reproduces by construction the fixed-order result, and no large spurious, higher-order, corrections arise in this region. The detailed matching formulas for the two schemes considered in our analysis are reported below.

We start by introducing a convenient notation for the perturbative expansion of the various ingredients. We define

$$
\sigma_{\mathrm{tot}}^{\mathrm{NNLO}}=\sum_{i=0}^{2} \sigma^{(i)}, \quad \Sigma^{\mathrm{NNLO}}\left(p_{\perp}\right)=\sigma^{(0)}+\sum_{i=1}^{2} \Sigma^{(i)}\left(p_{\perp}\right),
$$


where

$$
\Sigma^{(i)}\left(p_{\perp}\right)=\sigma^{(i)}+\bar{\Sigma}^{(i)}\left(p_{\perp}\right), \quad \bar{\Sigma}^{(i)}\left(p_{\perp}\right) \equiv-\int_{p_{\perp}}^{\infty} \mathrm{d} p_{\perp}^{\prime} \frac{\mathrm{d} \Sigma^{(i)}\left(p_{\perp}^{\prime}\right)}{\mathrm{d} p_{\perp}^{\prime}}
$$

Moreover, we denote the perturbative expansion of the resummed cross section $\Sigma^{\mathrm{NNLL}}$ as

$$
\mathcal{T}^{\mathrm{NNLO}}\left[\Sigma^{\mathrm{NNLL}}\left(p_{\perp}\right)\right]=\sigma^{(0)}+\sum_{i=1}^{2} \Sigma_{\mathrm{NNLL}}^{(i)}\left(p_{\perp}\right) .
$$

With this notation, the additive scheme of eq. (A.46) becomes (for simplicity we drop the explicit dependence on $p_{\perp}$ in the following)

$$
\Sigma_{\text {add }}=\Sigma^{\mathrm{NNLL}}+\left\{\sigma^{(1)}+\bar{\Sigma}^{(1)}-\Sigma_{\mathrm{NNLL}}^{(1)}\right\}+\left\{\sigma^{(2)}+\bar{\Sigma}^{(2)}-\Sigma_{\mathrm{NNLL}}^{(2)}\right\},
$$

where the three terms in curly brackets denote the NLO, NNLO and $\mathrm{N}^{3} \mathrm{LO}$ contributions to the matching, respectively.

For the multiplicative scheme we need to introduce the perturbative expansion of the asymptotic value $\Sigma_{\text {asym. }}^{\mathrm{NNLL}}$, defined in eq. (A.50). We write

$$
\Sigma_{\text {asym. }}^{\mathrm{NNLL}}=\sigma^{(0)}+\Sigma_{\text {asym. }}^{(1)} .
$$

With this notation the matching formula (A.51) reads

$$
\begin{aligned}
\Sigma_{\text {mult }}\left(p_{\perp}\right)=\frac{\Sigma^{\mathrm{NNLL}}}{\Sigma_{\text {asym. }}^{\mathrm{NNLL}}}\left[\sigma^{(0)}+\left\{\sigma^{(1)}+\bar{\Sigma}^{(1)}+\Sigma_{\text {asym. }}^{(1)}-\Sigma_{\mathrm{NNLL}}^{(1)}\right\}\right. \\
+\left\{\sigma^{(2)}+\bar{\Sigma}^{(2)}-\Sigma_{\text {NNLL }}^{(2)}+\frac{\Sigma_{\text {asym. }}^{(1)}}{\sigma^{(0)}}\left(\sigma^{(1)}+\bar{\Sigma}^{(1)}\right)+\frac{\left(\Sigma_{\text {NNLL }}^{(1)}\right)^{2}}{\sigma^{(0)}}\right. \\
\left.\left.+\frac{\Sigma_{\text {NNLL }}^{(1)}}{\sigma^{(0)}}\left(\sigma^{(1)}+\bar{\Sigma}^{(1)}+\Sigma_{\text {asym. }}^{(1)}\right)\right\}\right]
\end{aligned}
$$

where, as above, we grouped the terms entering at NLO, and NNLO within curly brackets.

Open Access. This article is distributed under the terms of the Creative Commons Attribution License (CC-BY 4.0), which permits any use, distribution and reproduction in any medium, provided the original author(s) and source are credited.

\section{References}

[1] ATLAS and CMS collaborations, Measurements of the Higgs boson production and decay rates and constraints on its couplings from a combined ATLAS and CMS analysis of the LHC pp collision data at $\sqrt{s}=7$ and $8 \mathrm{TeV}$, JHEP 08 (2016) 045 [arXiv:1606.02266] [INSPIRE].

[2] F. Bishara, U. Haisch, P.F. Monni and E. Re, Constraining light-quark Yukawa couplings from Higgs distributions, Phys. Rev. Lett. 118 (2017) 121801 [arXiv:1606.09253] [INSPIRE].

[3] Y. Soreq, H.X. Zhu and J. Zupan, Light quark Yukawa couplings from Higgs kinematics, JHEP 12 (2016) 045 [arXiv: 1606.09621] [INSPIRE]. 
[4] C. Anastasiou et al., Higgs Boson Gluon-Fusion Production in QCD at Three Loops, Phys. Rev. Lett. 114 (2015) 212001 [arXiv: 1503.06056] [INSPIRE].

[5] B. Mistlberger, Higgs boson production at hadron colliders at $N^{3} L O$ in $Q C D$, JHEP 05 (2018) 028 [arXiv:1802.00833] [INSPIRE].

[6] F. Dulat, B. Mistlberger and A. Pelloni, Differential Higgs production at $N^{3} L O$ beyond threshold, JHEP 01 (2018) 145 [arXiv:1710.03016] [INSPIRE].

[7] R. Boughezal et al., Higgs boson production in association with a jet at NNLO using jettiness subtraction, Phys. Lett. B 748 (2015) 5 [arXiv:1505.03893] [INSPIRE].

[8] R. Boughezal et al., Higgs boson production in association with a jet at next-to-next-to-leading order, Phys. Rev. Lett. 115 (2015) 082003 [arXiv:1504.07922] [INSPIRE].

[9] F. Caola, K. Melnikov and M. Schulze, Fiducial cross sections for Higgs boson production in association with a jet at next-to-next-to-leading order in QCD, Phys. Rev. D 92 (2015) 074032 [arXiv: 1508.02684] [inSPIRE].

[10] X. Chen, T. Gehrmann, E.W.N. Glover and M. Jaquier, Precise QCD predictions for the production of Higgs + jet final states, Phys. Lett. B 740 (2015) 147 [arXiv:1408.5325] [INSPIRE].

[11] X. Chen et al., NNLO QCD corrections to Higgs boson production at large transverse momentum, JHEP 10 (2016) 066 [arXiv:1607.08817] [INSPIRE].

[12] R.V. Harlander, H. Mantler, S. Marzani and K.J. Ozeren, Higgs production in gluon fusion at next-to-next-to-leading order QCD for finite top mass, Eur. Phys. J. C 66 (2010) 359 [arXiv: 0912.2104] [INSPIRE].

[13] A. Pak, M. Rogal and M. Steinhauser, Production of scalar and pseudo-scalar Higgs bosons to next-to-next-to-leading order at hadron colliders, JHEP 09 (2011) 088 [arXiv:1107.3391] [INSPIRE].

[14] R.V. Harlander and K.J. Ozeren, Finite top mass effects for hadronic Higgs production at next-to-next-to-leading order, JHEP 11 (2009) 088 [arXiv:0909.3420] [INSPIRE].

[15] R.V. Harlander, T. Neumann, K.J. Ozeren and M. Wiesemann, Top-mass effects in differential Higgs production through gluon fusion at order $\alpha_{s}^{4}$, JHEP 08 (2012) 139 [arXiv: 1206.0157] [INSPIRE].

[16] T. Neumann and M. Wiesemann, Finite top-mass effects in gluon-induced Higgs production with a jet-veto at NNLO, JHEP 11 (2014) 150 [arXiv: 1408.6836] [INSPIRE].

[17] T. Neumann and C. Williams, The Higgs boson at high $p_{T}$, Phys. Rev. D 95 (2017) 014004 [arXiv: 1609.00367] [INSPIRE].

[18] J.M. Lindert, K. Kudashkin, K. Melnikov and C. Wever, Higgs bosons with large transverse momentum at the LHC, Phys. Lett. B 782 (2018) 210 [arXiv:1801.08226] [inSPIRE].

[19] T. Neumann, NLO Higgs+jet at Large Transverse Momenta Including Top Quark Mass Effects, arXiv: 1802.02981 [INSPIRE].

[20] S.P. Jones, M. Kerner and G. Luisoni, Next-to-Leading-Order QCD Corrections to Higgs Boson Plus Jet Production with Full Top-Quark Mass Dependence, Phys. Rev. Lett. 120 (2018) 162001 [arXiv: 1802.00349] [INSPIRE].

[21] G. Bozzi, S. Catani, D. de Florian and M. Grazzini, Transverse-momentum resummation and the spectrum of the Higgs boson at the LHC, Nucl. Phys. B 737 (2006) 73 [hep-ph/0508068] [INSPIRE]. 
[22] D. de Florian, G. Ferrera, M. Grazzini and D. Tommasini, Transverse-momentum resummation: Higgs boson production at the Tevatron and the LHC, JHEP 11 (2011) 064 [arXiv: 1109.2109] [INSPIRE].

[23] D. de Florian, G. Ferrera, M. Grazzini and D. Tommasini, Higgs boson production at the LHC: transverse momentum resummation effects in the $H \rightarrow 2 \gamma, H \rightarrow W W \rightarrow l \nu l \nu$ and $H \rightarrow Z Z \rightarrow 4 l$ decay modes, JHEP 06 (2012) 132 [arXiv:1203.6321] [INSPIRE].

[24] T. Becher, M. Neubert and D. Wilhelm, Higgs-boson production at small transverse momentum, JHEP 05 (2013) 110 [arXiv:1212.2621] [INSPIRE].

[25] S. Catani and M. Grazzini, Higgs Boson Production at Hadron Colliders: Hard-Collinear Coefficients at the NNLO, Eur. Phys. J. C 72 (2012) 2013 [Erratum ibid. C 72 (2012) 2132] [arXiv: 1106.4652] [INSPIRE].

[26] T. Gehrmann, T. Luebbert and L.L. Yang, Calculation of the transverse parton distribution functions at next-to-next-to-leading order, JHEP 06 (2014) 155 [arXiv:1403.6451] [INSPIRE].

[27] Y. Li and H.X. Zhu, Bootstrapping rapidity anomalous dimensions for transverse-momentum resummation, Phys. Rev. Lett. 118 (2017) 022004 [arXiv:1604.01404] [INSPIRE].

[28] A.A. Vladimirov, Correspondence between soft and rapidity anomalous dimensions, Phys. Rev. Lett. 118 (2017) 062001 [arXiv: 1610.05791] [INSPIRE].

[29] W. Bizon et al., Momentum-space resummation for transverse observables and the Higgs $p_{\perp}$ at $N^{3} L L+N N L O$, JHEP 02 (2018) 108 [arXiv: 1705.09127] [INSPIRE].

[30] T. Becher, M. Neubert and L. Rothen, Factorization and $N^{3} L L_{p}+N N L O$ predictions for the Higgs cross section with a jet veto, JHEP 10 (2013) 125 [arXiv:1307.0025] [INSPIRE].

[31] W.-Y. Keung and F.J. Petriello, Electroweak and finite quark-mass effects on the Higgs boson transverse momentum distribution, Phys. Rev. D 80 (2009) 013007 [arXiv:0905.2775] [INSPIRE].

[32] K. Melnikov, L. Tancredi and C. Wever, Two-loop gg $\rightarrow$ Hg amplitude mediated by a nearly massless quark, JHEP 11 (2016) 104 [arXiv:1610.03747] [INSPIRE].

[33] K. Melnikov, L. Tancredi and C. Wever, Two-loop amplitudes for $q g \rightarrow H q$ and $q \bar{q} \rightarrow H g$ mediated by a nearly massless quark, Phys. Rev. D 95 (2017) 054012 [arXiv:1702.00426] [INSPIRE].

[34] M. Grazzini and H. Sargsyan, Heavy-quark mass effects in Higgs boson production at the LHC, JHEP 09 (2013) 129 [arXiv:1306.4581] [INSPIRE].

[35] A. Banfi, P.F. Monni and G. Zanderighi, Quark masses in Higgs production with a jet veto, JHEP 01 (2014) 097 [arXiv: 1308.4634] [INSPIRE].

[36] J.M. Lindert, K. Melnikov, L. Tancredi and C. Wever, Top-bottom interference effects in Higgs plus jet production at the LHC, Phys. Rev. Lett. 118 (2017) 252002 [arXiv: 1703.03886] [INSPIRE].

[37] G. Parisi and R. Petronzio, Small transverse momentum distributions in hard processes, Nucl. Phys. B 154 (1979) 427 [inSPIRE].

[38] H. Mantler and M. Wiesemann, Top- and bottom-mass effects in hadronic Higgs production at small transverse momenta through LO+NLL, Eur. Phys. J. C 73 (2013) 2467 [arXiv: 1210.8263] [INSPIRE].

[39] K. Hamilton, P. Nason and G. Zanderighi, Finite quark-mass effects in the NNLOPS POWHEG+MiNLO Higgs generator, JHEP 05 (2015) 140 [arXiv:1501.04637] [INSPIRE]. 
[40] E. Bagnaschi and A. Vicini, The Higgs transverse momentum distribution in gluon fusion as a multiscale problem, JHEP 01 (2016) 056 [arXiv: 1505.00735] [INSPIRE].

[41] E. Bagnaschi et al., Resummation ambiguities in the Higgs transverse-momentum spectrum in the Standard Model and beyond, JHEP 01 (2016) 090 [arXiv:1510.08850] [INSPIRE].

[42] K. Melnikov and A. Penin, On the light quark mass effects in Higgs boson production in gluon fusion, JHEP 05 (2016) 172 [arXiv: 1602.09020] [INSPIRE].

[43] T. Liu and A.A. Penin, High-energy limit of QCD beyond the Sudakov approximation, Phys. Rev. Lett. 119 (2017) 262001 [arXiv:1709.01092] [INSPIRE].

[44] F. Caola, S. Forte, S. Marzani, C. Muselli and G. Vita, The Higgs transverse momentum spectrum with finite quark masses beyond leading order, JHEP 08 (2016) 150 [arXiv: 1606.04100] [INSPIRE].

[45] U. Aglietti, R. Bonciani, G. Degrassi and A. Vicini, Analytic results for virtual QCD corrections to Higgs production and decay, JHEP 01 (2007) 021 [hep-ph/0611266] [INSPIRE].

[46] J. Butterworth et al., PDF 4 LHC recommendations for LHC Run II, J. Phys. G 43 (2016) 023001 [arXiv: 1510.03865] [INSPIRE].

[47] A. Buckley et al., LHAPDF6: parton density access in the LHC precision era, Eur. Phys. J. C 75 (2015) 132 [arXiv: 1412.7420] [INSPIRE].

[48] F. Cascioli, P. Maierhofer and S. Pozzorini, Scattering amplitudes with open loops, Phys. Rev. Lett. 108 (2012) 111601 [arXiv:1111.5206] [INSPIRE].

[49] F. Cascioli, J.M. Lindert, P. Maierhöfer and S. Pozzorini, The OpenLoops one-loop generator, publicly available at http://openloops.hepforge.org.

[50] A. Denner, S. Dittmaier and L. Hofer, Collier: a fortran-based Complex One-Loop LIbrary in Extended Regularizations, Comput. Phys. Commun. 212 (2017) 220 [arXiv:1604.06792] [INSPIRE].

[51] S. Alioli, P. Nason, C. Oleari and E. Re, A general framework for implementing NLO calculations in shower Monte Carlo programs: the POWHEG BOX, JHEP 06 (2010) 043 [arXiv: 1002.2581] [INSPIRE].

[52] T. Ježo and P. Nason, On the treatment of resonances in next-to-leading order calculations matched to a parton shower, JHEP 12 (2015) 065 [arXiv:1509.09071] [INSPIRE].

[53] K.G. Chetyrkin, J.H. Kuhn and M. Steinhauser, RunDec: a Mathematica package for running and decoupling of the strong coupling and quark masses, Comput. Phys. Commun. 133 (2000) 43 [hep-ph/0004189] [INSPIRE].

[54] F. Herren and M. Steinhauser, Version 3 of RunDec and CRunDec, Comput. Phys. Commun. 224 (2018) 333 [arXiv:1703.03751] [INSPIRE].

[55] P.F. Monni, E. Re and P. Torrielli, Higgs transverse-momentum resummation in direct space, Phys. Rev. Lett. 116 (2016) 242001 [arXiv: 1604.02191] [InSPIRE].

[56] A. Banfi, G.P. Salam and G. Zanderighi, Principles of general final-state resummation and automated implementation, JHEP 03 (2005) 073 [hep-ph/0407286] [INSPIRE].

[57] R. Harlander and P. Kant, Higgs production and decay: analytic results at next-to-leading order QCD, JHEP 12 (2005) 015 [hep-ph/0509189] [INSPIRE].

[58] C. Anastasiou et al., Two-loop amplitudes and master integrals for the production of a Higgs boson via a massive quark and a scalar-quark loop, JHEP 01 (2007) 082 [hep-ph/0611236] [INSPIRE]. 